\title{
Testing feasibility of scalar-tensor gravity by scale dependent mass and coupling to matter
}

\author{
D. F. Mota, \\ Institute of Theoretical Astrophysics, University of Oslo, 0315 Oslo, Norway \\ V. Salzano, \\ Fisika Teorikoaren eta Zientziaren Historia Saila, Zientzia eta Teknologia Fakultatea, \\ Euskal Herriko Unibertsitatea, 644 Posta Kutxatila, 48080 Bilbao, Spain \\ and S. Capozziello \\ Dipartimento di Scienze Fisiche, Universita' degli Studi di Napoli "Federico II" and INFN, \\ Sezione di Napoli, Complesso Universitario di Monte S. Angelo, Via Cinthia, Edificio N, 80126 Napoli, Italy
}

\begin{abstract}
We investigate whether there are any cosmological evidences for a scalar field with a mass and coupling to matter which change accordingly to the properties of the astrophysical system it "lives in", without directly focusing on the underlying mechanism that drives the scalar field scale-dependentproperties. We assume a Yukawa type of coupling between the field and matter and also that the scalar field mass grows with density, in order to overcome all gravity constraints within the solar system. We analyse three different gravitational systems assumed as "cosmological indicators": supernovae type Ia, low surface brightness spiral galaxies and clusters of galaxies. Results show that: $a$. a quite good fit to the rotation curves of low surface brightness galaxies only using visible stellar and gas mass components is obtained; $b$. a scalar field can fairly well reproduce the matter profile in clusters of galaxies, estimated by X-ray observations and without the need of any additional dark matter; $c$. there is an intrinsic difficulty in extracting information about the possibility of a scale-dependent massive scalar field (or more generally about a varying gravitational constant) from supernovae type Ia.
\end{abstract}

Keywords: gravitation - dark matter - dark energy - galaxies: clusters : intracluster medium - galaxies: dwarf - galaxies : kinematics and dynamics

\section{INTRODUCTION}

Present cosmological and astrophysical observations clearly depict a universe dominated by "dark" components, being it made by dark matter for $25 \%$ and by dark energy for $71 \%$, while ordinary baryonic matter contributes for only the remaining $4 \%$ [50].

Dark matter has a long history, being "introduced" by 92]: for solving the problems of high mass-to-light ratios of galaxy clusters and of the rotation curves of spiral galaxies. Later on, several versions of the the so-called cold dark matter model (CDM) have been built starting from the assumption that a large amount of non-baryonic matter, matter non-interacting with the electromagnetic radiation but only detectable by its gravitational interaction with visible matter, could account for the observations in the framework of the standard Newtonian dynamics. But even if its clustering and distribution properties are fairly well known at every scale (see [56] for the most used model), its nature is unknown, up to now, at a fundamental level.

Dark energy has a more recent history: it was "introduced" about ten years ago while reconstructing the Hubble diagram of Type Ia Supernovae (SNeIa) observations, from which it was deduced that the universe is now accelerating [1, 25, 67, 71]. While growing the quantity of available cosmological data (measurements of cluster properties as the mass, the correlation func- tion and the evolution with redshift of their abundance [3, 4, 36, 88]; the already mentioned Hubble diagram of SNeIa; the optical surveys of large scale structure [27, 35, 68]; the anisotropies in the cosmic microwave background [29, 50, 78]; the cosmic shear measured from weak lensing surveys [69, 87] and the Lyman - $\alpha$ forest absorption [28, 53]), more evidences towards a spatially flat universe with a subcritical matter content and undergoing a phase of accelerated expansion have been collected. Despite of the existence of universe acceleration has been clearly undisclosed, the nature and the fundamental properties of the underneath physical mechanism remain essentially unknown notwithstanding the great theoretical efforts made up to now. By simply adding a constant to the dynamical equations of universe, the cosmological constant $\Lambda$ [16, 72], in the context of CDM model, it was defined a new model, the $\Lambda \mathrm{CDM}$, which quickly became the consensus model because it provides a good fit to most of the data [73, 77, 82] giving a reliable snapshot of the today observed universe. Nevertheless, it is affected by serious theoretical shortcomings that have motivated the search for more general and alternative candidates generically referred to as dark energy. Such models range from scalar fields rolling down self interaction potentials to phantom fields, from phenomenological unified models of dark energy and dark matter to alternative gravity theories [17, 20, 26, 65, 66].

In the last three decades, scalar fields have played an important role in both cosmology and particle physics 
[5, 52]. Scalar fields have been postulated as means to explain the early and late time acceleration of the universe. However, it is almost always the case that such fields interact with standard matter: either due to a direct Lagrangian coupling or indirectly through a coupling to the Ricci Scalar or as the result of quantum loop corrections. Both for inflation in the early universe and for dark energy, such couplings can lead to problems. In inflation, for example, couplings might destroy the flatness of the potential needed to drive a period of inflation. If there are scalar fields which permeate the universe today and have non-zero couplings to matter, then they would induce an additional force in nature. If the scalar field self-interactions are negligible, then the experimental bounds on such a field are very strong: either the couplings to matter are much smaller than gravity, or the scalar fields are very heavy, so that they mediate a short-ranged interaction.

A certain class of theories have been proposed, in which the scalar field properties depend on the environment: its mass depends on the local environmental density. These are the so called Chameleon Field Theories, proposed by [48], that employs a combination of selfinteraction and couplings to matter of the scalar field to avoid the most restrictive of the current gravity bounds. In these models a scalar field couples to matter with gravitational strength, in harmony with general expectations from string theory, whilst, at the same time, remaining relatively light on cosmological scales. It was found that local gravity constraints are (roughly) satisfied as long as the mass-scale of the potential satisfies $M \lesssim(1 \mathrm{~mm})^{-1}$. This coincides with the scale associated with the late time acceleration of the universe, and it is surprising that it should come from local experiments. Chameleon models have been subject to many studies, from laboratory experiments up to cosmological probes 8 - 10, 33, 40, 43, 46, 57 62, 83 85]. Lately, chameleons models where also studied in the context of Modified Gravity, in particular the so called $f(R)$-gravity [11, 19, 39]. In such a case, good results of general relativity, according to the standard probes, are reproduced at local scales while dark energy (accelerating behavior) and dark matter (dynamical clustering) effects are reproduced at larger scales. The major issue is to select suitable $f(R)$-models capable of matching the density profiles at the various gravitational scales, as discussed e.g. in [19].

Chameleon fields evade tight gravity constraints via the so called chameleon mechanism: it consists on a fieldgenerated force, a sort of fifth force, to become shortranged in highly dense regions, and long-ranged in low density regions. Such feature would imply that at different astrophysical scales the fifth force felt by matter would be suppressed or enhanced according to the local astrophysical density.

In this work we investigate whether there is evidence for a coupling between baryonic matter and a massive scalar field which could mimic and replace the con- tributions of a possible dark matter component. In particular, motivated by chameleon models, we aim to verify if it is possible to observationally detect a scalar field whose mass (or, equivalently, interaction length) and coupling may change with scale, matching different astrophysical observations. In this case, by astrophysical observations, we mean data from SNeIa, low surface brightness (LSB) dwarf galaxies and, finally, clusters of galaxies. The range of scales is very wide and we are adopting photometric and spectroscopic data to probe the mechanism at different redshifts.

We want to stress here that we are just interested to investigate whether the data would favor a model where baryons may be coupled to a scalar field whose mass and coupling change with scale. If such a change is due to a chameleon mechanism (associated to a local-density variation) or due to some other mechanism, that is not the main concern in this article and so we will not compute specific predictions for any particular model.

Chameleon models are highly non linear so that, in principle, no superposition principle for such a non-linear field would be possible. Due to that, it is extremely difficult to build the gravitational potential of an extended astrophysical system as the ones we analyse in this work. Fortunately, we are not specifically investigating chameleon models; we are here studying a general coupled scalar field model and asking the data what are the preferred values of its mass and coupling at different scales. In order to compute an extended gravitational potential (in a non-linear case as the chameleon model would be), we would need to properly study the nonlinear regime of structure formation within this type of models. But this is well beyond the scope of this article. In spite of being of utmost importance to set up N-body simulations with heavy scalar field models, such task is extremely complex. In fact up to nowadays most of the N-body simulations assume light scalar fields which do not cluster at small scales. That is obviously not the case of chameleon fields and such investigation is not considered in the present paper.

The article is organized as follows: In $\S$ (II) we give a brief but exhaustive summary of all the main properties of the scalar field theory. In $\S$ (III) we accurately describe the used astrophysical and cosmological data and the theoretical model defined for any of them. In $\S$ (IV) we expose our results with a discussion on their implications for a more general and comprehensive theory of gravity. Conclusions are drawn in $\S(\mathbb{V})$

\section{THE SCALAR FIELD THEORY}

A general action governing the dynamics of the chameleon (scalar) field $\phi$ can be of the form:

$$
S=\int \mathrm{d}^{4} x \sqrt{-g}\left\{\frac{M_{P l}^{2}}{2} \mathcal{R}-\frac{1}{2}(\partial \phi)^{2}-V(\phi)\right\}
$$



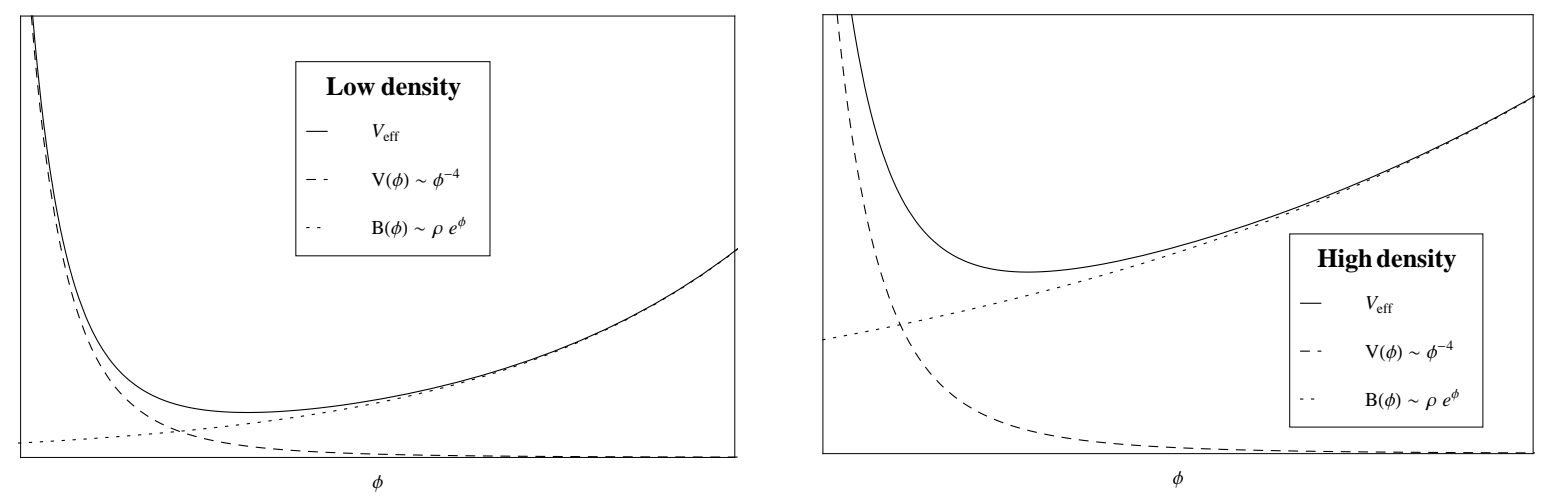

Figure 1. Chameleon potential in low and high local density environment.

$$
-\int \mathrm{d}^{4} x \mathcal{L}_{m}\left(\psi_{m}^{(i)}, g_{\mu \nu}^{i}\right),
$$

where $g$ is the determinant of the metric $g_{\mu \nu}, \mathcal{R}$ is the Ricci scalar, $\psi_{m}^{(i)}$ are the various matter fields and $\mathcal{L}_{m}$ is the Lagrangian density of ordinary matter.

In the expression for the reduced Planck Mass, $M_{P l} \equiv$ $\left(8 \pi G_{*}\right)^{-1 / 2}, G_{*}$ is the bare gravitational constant and differs from the usually measured one.

This can be better understood if we consider the most general case of a scalar-tensor model, whose action (Eq. [1 is a particular case of this) is 37]:

$$
\begin{aligned}
S & =\frac{1}{16 \pi G_{*}} \int \mathrm{d}^{4} x \sqrt{-g}\left\{F(\phi) \mathcal{R}-Z(\phi)(\partial \phi)^{2}+\right. \\
& -V(\phi)\}-\int d^{4} x \mathcal{L}_{m}\left(\psi_{m}^{(i)}, g_{\mu \nu}^{i}\right) .
\end{aligned}
$$

$f(R)$-gravity models too can be enclosed in this general case since it is straightforward to show that, by suitable manipulations, they are a subclass of Eq. (2); see for example [20]. From this action one can naively define Newton's gravitational constant as:

$$
G_{N} \doteq \frac{G_{*}}{F},
$$

but $G_{N}$ has not the same physical meaning as Newton's gravitational constant in general relativity. The actual Newtonian force measured in a Cavendish-type experiment between two test masses will experience an effective coupling constant

$$
G_{e f f}=\frac{G_{*}}{F}\{1+\alpha(\phi)\} \doteq G_{N}\{1+\alpha(\phi)\},
$$

where the term $G_{*} / F$ is due to the (average) exchange of gravitons between the two bodies, while $G_{*} / F \cdot \alpha(\phi)$ comes from the exchange of a scalar particle between them, with the analytical expression for $\alpha$ depending on the particular scalar theory one considers. It is also clear from this expression, that what in general relativity is a true constant, now becomes a possible function of time and radius, so the use of the term "constant" is quite inappropriate. When we will speak about a varying gravitational constant we always refer to the effective scalar field gravitational constant expression.

The key ingredient in studying the cosmological dynamics of a field, when it supports a chameleon mechanism, is that the scalar field feels both a potential, $V(\phi)$, and a coupling to matter depending on $\rho$, the local density of matter, and on the coupling constant, $\beta$. At the end the field dynamics is governed by an effective potential,

$$
V_{e f f}(\phi)=V(\phi)+\sum_{i} \rho_{i} e^{\beta_{i} \phi / M_{P l}}
$$

If $V(\phi)$ is a run-away potential and $\beta_{i}>0$, the effective potential has a minimum at $\phi_{\min }$ satisfying the condition:

$$
V_{, \phi}\left(\phi_{\min }\right)+\sum_{i} \frac{\beta}{M_{P l}} \rho_{i} e^{\beta_{i} \phi / M_{P l}}=0
$$

and the effective mass of the field of small perturbations about the minimum is

$$
m^{2}=V_{, \phi \phi}^{e f f}=V_{, \phi \phi}\left(\phi_{m i n}\right)+\sum_{i} \frac{\beta^{2}}{M_{P l}^{2}} \rho_{i} e^{\beta_{i} \phi / M_{P l}} .
$$

So the effective potential depends on the matter density and both the minimum in potential and the mass of the scalar field are function of the local density, as shown in Fig. (11). As density increases, the minimum in potential shifts to smaller values of the field and the mass of small fluctuations increases. This last property, in particular, makes the chameleon field able to satisfy the constraints from laboratory tests of the principle of equivalence, because in high densities environment, such as terrestrial laboratories, the field can be heavy enough so to evade them.

It must be stressed, however, that even with such a mechanism, it is very difficult to build a theory with a late time cosmology observationally indistinguishable from the standard $\Lambda \mathrm{CDM}$ model. 
Another interesting consequence of this model comes out when considering linear perturbations of matter and their related equation. In the most general case of a scalar-tensor theory [7] we have:

$$
\delta_{c}^{\prime \prime}+a H \delta_{c}^{\prime}=\frac{3}{2} a^{2} H^{2}\left[1+\frac{2 \beta^{2}}{1+a^{2} V_{, \phi \phi} / k^{2}}\right],
$$

where $a$ is the scale factor, $H$ is the Hubble function, $\delta_{c}=\delta\left(\rho_{m} e^{\beta \phi / M_{P l}}\right)$ is the matter density contrast and, if the field is at the minimum, its mass is $m^{2}=V_{, \phi \phi}$. The quantity in brackets can be interpreted as the expression for the effective gravitational constant within the context of massive scalar field models [42]:

$$
G_{e f f}(a ; \beta, m ; k)=G_{N}\left(1+2 \beta^{2} \frac{\frac{k^{2}}{a^{2} m^{2}}}{1+\frac{k^{2}}{a^{2} m^{2}}}\right)
$$

In particular the term proportional to $\mathrm{m}^{2}$ results from the scalar field-mediated force, which is negligible if the physical length scale of the perturbation is much larger than the range of the chameleon-mediated force, namely, if $a / k \gg m^{-1}$. In this case the left hand side of Eq. (8) is well approximated by $3 a^{2} H^{2} \delta_{c} / 2$ and the matter fluctuations grow as in general relativity.

Anyway chameleon theories (i.e. based on Eq. 5) do not behave like linear theories (as anticipated in the introductory section) of massive scalar fields when massive bodies are involved. Varying the action Eq. (1) with respect to the chameleon (scalar) field $\phi$ in a spherically symmetric spacetime gives:

$$
\frac{\mathrm{d}^{2} \phi}{\mathrm{d} r^{2}}+\frac{2}{r} \frac{\mathrm{d} \phi}{\mathrm{d} r}=\frac{\mathrm{d} V_{e f f}}{\mathrm{~d} \phi},
$$

because of the effective potential for $\phi$ changes in different density environments, this differential equation is highly non-linear. These non-linear features have been studied in [48], where it was found that for a spherically symmetric object of mass $M_{c}$ and radius $R_{c}$ surrounded by a gas of asymptotic density $\rho_{\infty}$, the profile of the field is governed by the so-called "thin-shell" parameter,

$$
\Delta=\frac{\left|\phi_{\min }^{\infty}-\phi_{\min }^{c}\right|}{6 \beta M_{P l} \Phi_{c}}
$$

where $\phi_{\min }^{\infty}$ and $\phi_{\min }^{c}$ are the minima of the effective potential outside and inside the object respectively and $\Phi_{c}=M_{c} / 8 \pi M_{P l}^{2} R_{c}$ is the Newtonian potential at the surface of the object. Thus $\Delta$ is the ratio of the difference in $\phi$ potential to the Newtonian potential and quantifies how perturbing the object is for the $\phi$ field. If $\Delta$ is large, which happens for small objects, then the external (to the object) profile of $\phi$ is the usual Yukawa profile,

$$
\phi(r)=-\left(\frac{\beta}{4 \pi M_{P l}}\right) \frac{M_{c} \exp ^{-m_{\infty}}}{r}+\phi_{m i n}^{\infty},
$$

where $m_{\infty}$ is the mass of the scalar field. For large and compact objects, $\Delta$ is small and the Yukawa profile is suppressed by a factor of $\Delta$. Thus the term "thin-shell" comes from the fact that only a portion of such a "thin-shell" contributes to the external Yukawa profile. A discussion of this issue in the framework of $f(R)$-gravity is in [19].

In summary the main ingredients of our model are:

- a massive scalar field coupled with ordinary observable baryonic mass;

- its mass $m$, or its interaction length $L \propto m^{-1}$;

- its coupling constant with baryonic mass $\beta$

\section{A. Modified gravitational potential}

Taking the inverse Fourier transform of Eq. (9) it is straightforward to obtain the corresponding expression of the gravitational potential for a point mass distribution, $\psi(r)$. Remembering that a potential $\propto \frac{1}{r}$ in real space yields a $k^{-2}$ term in Fourier space, we can recognize in Eq. (9) the point-like gravitational potential per unit mass:

$$
\begin{aligned}
\psi(r) & =-\frac{G}{r}\left(1+2 \beta^{2} e^{-m r}\right)= \\
& =-\frac{G}{r}\left(1+2 \beta^{2} e^{-r / L}\right),
\end{aligned}
$$

where $m$ is the mass of the scalar field, $L \propto m^{-1}$ is the interaction range of the modified gravitational potential, i.e. the length where the scalar field is effective, and $\beta$ still being the coupling constant between matter and the scalar field. The gravitational potential given in Eq. (13) is a point-like one, so that we have to generalize it to extended systems if we want to use it for clusters of galaxies and LSB galaxies. As we will discuss later, we are going to model galaxy clusters as spherically symmetric systems: we simply consider the system composed by many infinitesimal mass elements each one contributing with a point-like gravitational potential. Then, summing up all terms, namely integrating them on a spherical volume, we obtain a suitable potential. Specifically, we have to solve the integral:

$$
\Psi(r)=\int_{0}^{\infty} r^{\prime 2} d r^{\prime} \int_{0}^{\pi} \sin \theta^{\prime} d \theta^{\prime} \int_{0}^{2 \pi} d \omega^{\prime} \psi\left(r^{\prime}\right) .
$$

We make explicit that with an abuse of notation we are writing inside the point-like potential $r^{\prime}$, while it should be replaced by $\left|\overrightarrow{\mathbf{x}}-\overrightarrow{\mathbf{x}}^{\prime}\right|=\left(r^{2}+r^{\prime 2}-2 r r^{\prime} \cos \theta^{\prime}\right)^{1 / 2}$.

The point-like potential can be split in two terms. The Newtonian component is:

$$
\psi_{N}(r)=-\frac{G M}{r},
$$

and its extended integral is the well-known expression:

$$
\Psi_{N}(r)=-\frac{G M(<r)}{r},
$$


where $M(<r)$ is the mass enclosed in a sphere with radius $r$. The correction term coming from the scalar field is:

$$
\psi_{C}(r)=-\frac{G M}{r}\left(2 \beta^{2} e^{-\frac{r}{L}}\right)
$$

from the integration of the angular part, we have:

$$
\begin{aligned}
\Psi_{C}(r) & =-2 \pi G\left(2 \beta^{2} L\right) \int_{0}^{\infty} \mathrm{d} r^{\prime} r^{\prime} \rho\left(r^{\prime}\right) . \\
& \cdot \frac{e^{-\frac{\left|r-r^{\prime}\right|}{L}}-e^{-\frac{\left|r+r^{\prime}\right|}{L}}}{r}
\end{aligned}
$$

The radial integral is numerically estimated once the mass density is given. A fundamental difference between such a term and the Newtonian one is that while in the latter the matter outside the spherical shell of radius $r$ does not contribute to the potential, in the former external matter takes part to the integration procedure, even if its contribution is really negligible in most cases.

At the end, the total potential of the spherical mass distribution will be:

$$
\Psi(r)=\Psi_{N}(r)+\Psi_{C}(r)
$$

As we will show below, for our purpose we need the gravitational potential derivative with respect to the variable $r$; this may not be evaluated analytically so we estimate it numerically, once we have given an expression for the mass density $\rho(r)$. While the Newtonian term gives the simple expression:

$$
\frac{\mathrm{d} \Phi_{N}}{\mathrm{~d} r}(r)=\frac{G M(<r)}{r^{2}}
$$

the derivative of the corrective potential term is more involved. We do not give it explicitly for sake of brevity, but only remind that it is an integral-function of the form

$$
\mathcal{F}\left(r, r^{\prime}\right)=\int_{\alpha(r)}^{\beta(r)} d r^{\prime} f\left(r, r^{\prime}\right)
$$

from it one has:

$$
\begin{aligned}
\frac{\mathrm{d} \mathcal{F}\left(r, r^{\prime}\right)}{\mathrm{d} r} & =\int_{\alpha(r)}^{\beta(r)} d r^{\prime} \frac{\mathrm{d} f\left(r, r^{\prime}\right)}{\mathrm{d} r}-f(r, \alpha(r)) \frac{\mathrm{d} \alpha}{\mathrm{d} r}(r)+ \\
& +f(r, \beta(r)) \frac{\mathrm{d} \beta}{\mathrm{d} r}(r) .
\end{aligned}
$$

Such an expression is numerically derived once the integration extremes are given.

For spiral galaxies, we have the same theoretical apparatus but a different geometric configuration. Matter in spiral galaxies is generally modeled as distributed in a thin axis-symmetric disk, so that the extended gravitational potential is given by:

$$
\Psi(r, z)=\int_{0}^{\infty} R^{\prime} \mathrm{d} R^{\prime} \int_{0}^{2 \pi} \mathrm{d} \omega^{\prime} \psi\left(R^{\prime}, z^{\prime}\right) .
$$

Even in this case, for being more exact, the couple of variables $\left(R^{\prime}, z^{\prime}\right)$ inside the point-like potential should be replaced by $\left|\overrightarrow{\mathbf{x}}-\overrightarrow{\mathbf{x}}^{\prime}\right|=\left(R^{2}+R^{\prime 2}-2 R R^{\prime} \cos \omega^{\prime}+\left(z-z^{\prime}\right)^{2}\right)^{1 / 2}$. Once the gravitational potential is given, the rotation curve for the disk can be easily computed starting from the relation [6]:

$$
v_{c}^{2}=\left.R \frac{\mathrm{d} \Psi(R, z)}{\mathrm{d} R}\right|_{z=0}
$$

It is important to underline that both in the clusters of galaxies case and in the LSB galaxies one, we have to perform derivatives with respect of the distance from the center of any system, $r$, of the numerically derived gravitational potential. To be completely rigorous we should add a term to all the previously written relations, coming from the derivative of the function $\beta(\rho) \sim \beta(r)$. In our scalar-field approach we have treated the coupling parameter as a constant at all, while in the definition of the chameleon mechanism it is also possible and not trivial that it may depend on the local density of the gravitational system one is going to consider. At the same time, we do not know what is the possible analytical behavior of this quantity or, better: it is one of our purposes trying to reconstruct it. It is also evident that if we want to use previous relations in the form we have shown before, we are implicitly assuming that $\beta$ can satisfy two different scenarios: $a$. it is really constant, and in this case one expects not to detect any change in it when comparing different gravitational scales; or (as we will shown to be our case) $b$. it is a function of the gravitational scale, but its derivative is supposed to be negligible, i.e. $\mathrm{d} \beta / \mathrm{d} r \approx 0$.

\section{B. Modified distance modulus}

In 7] the Friedmann equation is derived from the action governing the dynamics of the chameleon field $\phi$ in the Jordan frame:

$$
3 H^{2} M_{P l}^{2}=\rho_{m} e^{\beta \phi / M_{P l}}+\frac{1}{2} \dot{\phi}^{2}+V(\phi)+\rho_{r},
$$

with contribution from matter, radiation and the scalar field. Making explicit the expression for the Planck mass, Eq. (25) becomes:

$$
3 H^{2}=8 \pi G_{*}\left[\rho_{m} e^{\beta \phi / M_{P l}}+\left(\frac{1}{2} \dot{\phi}^{2}+V(\phi)\right)+\rho_{r}\right] .
$$

We can show that this equation in the chameleon case easily converts in the most general expression for a given scalar field $\phi$.

If we assume the chameleon field $\phi$ is in the minimum of the effective potential from the early stages of the universe on, then we have $\frac{\phi}{M_{P L}} \ll 1$ [42] during the subsequent evolution until today. This also means that $e^{\beta \phi / M_{P L}}=1$ to very high accuracy so it will disappear from equations and does not have to be considered 
here. Then, considering that the function $F$ appearing in Eq. (3) is equal to unity in the scalar field case, we also have $G_{N}=G_{*}$. At the end the Friedmann equation is completely equal to the usual expression:

$$
3 H^{2}=8 \pi G_{N}\left[\rho_{m}+\left(\frac{1}{2} \dot{\phi}^{2}+V(\phi)\right)+\rho_{r}\right],
$$

and finally, avoiding radiation contribution while suffix $S c$ corresponds to the scalar field:

$$
h^{2}(z)=\Omega_{m, 0}(1+z)^{3}+\Omega_{S c, 0} \epsilon(z),
$$

where:

$$
\begin{aligned}
h(z) & \doteq \frac{H(z)}{H_{0}} \\
\Omega_{m, 0} & \doteq \frac{8 \pi G_{N}}{3 H_{0}^{2}} \rho_{m, 0} \\
\Omega_{S c, 0} & \left.\doteq \frac{8 \pi G_{N}}{3 H_{0}^{2}}\left(\frac{1}{2} \dot{\phi}^{2}+V \phi\right)\right|_{z=0}=\frac{8 \pi G_{N}}{3 H_{0}^{2}} \rho_{S c, 0}
\end{aligned}
$$

The function $\epsilon(z)$ is unknown; but one knows (assumes) that the scalar field works like a cosmological constant on cosmological scales, so we may choose it to be constant in redshift or one can use more general and extended models as the Chevallier-Polarski-Linder (CPL) parametrization [24, 51] usually used to phenomenologically describe dark energy fluids.

Even if we do not have any possibility to discriminate between $\Lambda \mathrm{CDM}$ and a scalar field scenario only by using $h(z)$, we have a discriminating tool in the distance modulus, the main observable quantity derivable from SNeIa observations, modified from the usual expression by assuming that the gravitational constant can vary with time:

$$
\begin{aligned}
\mu(z ; \beta, m ; k) & =5 \log \left((1+z) \int_{0}^{z} \frac{\mathrm{d} z}{h(z)}\right)+\mu_{0} \\
& +\frac{15}{4} \log \frac{G_{e f f}(z ; \beta, m ; k)}{G_{e f f}(0 ; \beta, m ; k)} .
\end{aligned}
$$

In this expression there is an additional term made of with the ratio between the value of effective gravitational constant at any redshift and the same quantity evaluated at the present $(z=0)$. As accurately described in [70], a time-varying gravitational constant can affect light curves from SNeIa by changing both the thermonuclear energy release, since the luminosity at the maximum in the light curve is proportional to the mass of synthesized nickel, and the time scale of stellar explosion. This means that by using Eq. (30) we are going to test the scalar field mechanism on cosmological scales, in particular considering the consequent role of a possible variation of the effective gravitational constant in the Universe acceleration rate history.

In the scalar field theory one has an analytical expression for the effective gravitational constant, i.e. Eq. (9), which we have modified in the following one for uniforming all the results:

$$
G_{e f f}(z ; \beta, L ; \lambda)=G_{N}\left(1+2 \beta^{2} \frac{(1+z)^{2} L^{2}}{(1+z)^{2} L^{2}+\lambda^{2}}\right),
$$

which depends on the following variables:

- the redshift $z(a=1 /(1+z))$;

- the wavelength $k$ (or the length $\lambda \propto k^{-1}$ ), which one could fix or vary on a grid;

- the intrinsic model parameters, i.e., the coupling constant $\beta$ and the interaction length $L \propto m^{-1}$, which can be constrained with a fitting procedure.

\section{OBSERVATIONAL DATA}

We are going to test the scalar field mechanism on three different scale ranges:

- on cosmological scales, by means of supernovae luminosity distance;

- on a Mpc-astrophysical scale, using mass profiles of clusters of galaxies;

- on a kpc-astrophysical scale, analyzing rotation curves from spiral galaxies.

For any of them, we have found out the necessary sample data in literature.

\section{A. Cosmological scale: Supernovae}

SNeIa are useful because of the possibility to easily modify the expression of distance modulus for more general theories with a varying gravitational constant (as in the scalar field case). Moreover, the distance modulus is the main observable quantity derivable from this kind of astrophysical objects. Adding to that, the possibility of using data ranging up to redshift values much larger than those ones from galaxies or clusters of galaxies $(z \approx 2)$ makes us possible to test and verify a possible temporal variation of the gravitational constant (if there is any) and so a possible alternative gravity scenario. It is interesting to underline that with the modified expression of the distance modulus we can also verify the coexistence, at the same time, of both dark energy and dark matter, both explained as different consequences on different scales of the same unified context, namely, the scalar field. In fact, in the expression for the distance modulus we are going to describe in next sections, we employ both a term with a dark energy-modeled fluid, coming out nothing else that from the effective behavior of the scalar field on cosmological scales (which does not differ much from the cosmological constant behavior) and a 
term acting as a dark matter-modeled component, coming out from the scalar field working on gravitational scales smaller than the cosmological one. In this sense, we have also explored how the scalar field process can mimic dark matter profiles in clusters and spiral galaxies.

We use the Constitution sample described in [45], which is a data set obtained by combining the Union data set by [49] with new 90 nearby objects from the CfA3 release described in [4].

The Union SNeIa compilation is a data set of lowredshift nearby-Hubble-flow SNeIa and is built with new analysis procedures for working with several heterogeneous SNeIa compilations. It includes 13 independent sets with SNe from the SCP, High-z Supernovae Search (HZSNS) team, Supernovae Legacy Survey and ESSENCE Survey, the older data sets, as well as the recently extended data set of distant supernovae observed with HST. After various selection cuts were applied in order to create a homogeneous and high-signal-to-noise data set, we have final 307 SNeIa events distributed over the redshift interval $0.15 \leq z \leq 1.55$.

The CfA3 data set is originally made of 185 multiband optical SNeIa light curves taken at the F.L. Whipple Observatory of the Harvard-Smithsonian Center for Astrophysics (CfA); 90 of the original 185 objects pass the quality cuts of [49] and are added to the Union data set to form the Constitution one.

The statistical analysis of Constitution SNeIa sample rests on the definition of the distance modulus given in Eq. (30). The best fits were obtained by minimizing the quantity

$$
\chi_{\mathrm{SN}}^{2}\left(\mu_{0}, \lambda,\left\{\theta_{i}\right\}\right)=\sum_{j=1}^{\mathcal{N}} \frac{\left(\mu\left(z_{j} ; \mu_{0}, \lambda,\left\{\theta_{i}\right)\right\}-\mu_{o b s}\left(z_{j}\right)\right)^{2}}{\sigma_{\mu}^{2}\left(z_{j}\right)}
$$

where $\mathcal{N}=397$ is the number of observed SNeIa, $\mu$ is the distance modulus (the observed, $\mu_{o b s}$, and the theoretical one, $\mu\left(z_{j} ; \mu_{0}, \lambda,\left\{\theta_{i}\right)\right), \sigma_{\mu}^{2}$ are the measurement variances and $\left\{\theta_{i}\right\}=\{\beta, L\}$ is the parameters theory vector. The nuisance parameter $\mu_{0}$ encodes the Hubble parameter and the absolute magnitude $M$, and has to be marginalized over. Giving the heterogeneous origin of the Constitution data set, and the procedures described in [49] and [45] for reducing data, we have worked with an alternative version Eq. (32), which consists in minimizing the quantity

$$
\tilde{\chi}_{\text {SN }}^{2}\left(\left\{\theta_{i}\right\}\right)=c_{1}-\frac{c_{2}^{2}}{c_{3}}
$$

with respect to the other parameters. Here

$$
\begin{aligned}
c_{1} & =\sum_{j=1}^{\mathcal{N}} \frac{\left(\mu\left(z_{j} ; \mu_{0}=0,\left\{\theta_{i}\right)\right\}-\mu_{o b s}\left(z_{j}\right)\right)^{2}}{\sigma_{\mu}^{2}\left(z_{j}\right)}, \\
c_{2} & =\sum_{j=1}^{\mathcal{N}} \frac{\left(\mu\left(z_{j} ; \mu_{0}=0,\left\{\theta_{i}\right)\right\}-\mu_{o b s}\left(z_{j}\right)\right)}{\sigma_{\mu}^{2}\left(z_{j}\right)},
\end{aligned}
$$

$$
c_{3}=\sum_{j=1}^{\mathcal{N}} \frac{1}{\sigma_{\mu}^{2}\left(z_{j}\right)} .
$$

It is trivial to see that $\tilde{\chi}_{S N}^{2}$ is just a version of $\chi_{S N}^{2}$, minimized with respect to $\mu_{0}$. To that end it suffices to notice that

$$
\chi_{\mathrm{SN}}^{2}\left(\mu_{0}, \lambda,\left\{\theta_{i}\right\}\right)=c_{1}-2 c_{2} \mu_{0}+c_{3} \mu_{0}^{2}
$$

which clearly becomes minimum for $\mu_{0}=c_{2} / c_{3}$, and so we can see $\tilde{\chi}_{\text {SN }}^{2} \equiv \chi_{\text {SN }}^{2}\left(\mu_{0}=0, \lambda,\left\{\theta_{i}\right\}\right)$. Furthermore, one can check that the difference between $\chi_{S N}^{2}$ and $\tilde{\chi}_{S N}^{2}$ is negligible.

We minimize the $\chi$-square using the Markov Chains Monte Carlo Method (MCMC) and testing their convergence with the method described by [34]. The $i \sigma$ confidence levels are easily estimated deriving them from the final samples, using the 15.87-th and 84.13-th quartiles (which define the $68 \%$ confidence interval) for $i=1$, the 2.28-th and 97.72-th quartiles (which define the $95 \%$ confidence interval) for $i=2$ and the 0.13 -th and 99.87-th quantiles (which define the $99 \%$ confidence interval) for $i=3$.

\section{B. Galaxy Cluster Sample}

Clusters of galaxies are uniquely useful tracer of cosmological evolution and so ineludible tests in the field of alternative gravities other than general relativity [91]. They are fundamental tracers for two main features. First of all, they are the largest gravitational objects whose masses can be adequately measured, and the largest objects to have undergone gravitational relaxation and entered into virial equilibrium. Second, clusters are essentially "closed boxes" that retain all their gaseous mass content because their gravitational wells are much deep. The most accepted paradigm is that clusters of galaxies are mostly made of collisionless cold dark matter particles (CDM model) and are virialized systems from scale-free Gaussian initial density perturbations. The CDM paradigm and the numerical simulations make clear predictions for the structure of clusters of galaxies; comparisons of these predictions with the results of high-quality observations is a necessary consistent check and any significant deviation can place important constraints on their theoretical model but also on cosmological models and, as in our case, on the exploration of different gravity theories.

The formalism described in $\S \llbracket$ can be applied to a sample of 12 galaxy clusters. We shall use the cluster sample studied in [89, 90] which consists of 13 lowredshift clusters spanning a temperature range $0.7 \div$ $9.0 \mathrm{keV}$ derived from high quality Chandra archival data. In all these clusters, the surface brightness and the gas temperature profiles are measured out to large radii, so that mass estimates can be extended up to $r_{500}$ or beyond. 
Clusters of galaxies are generally considered self-bound gravitational systems with spherical symmetry and in hydrostatic equilibrium if virialized. The last two hypotheses are still widely used, despite of the fact that it has been widely proved that most clusters show more complex morphologies and/or signs of strong interactions or dynamical activity, especially in their innermost regions [23, 32].

Under the hypothesis of spherical symmetry in hydrostatic equilibrium, the structure equation can be derived from the collisionless Boltzmann equation

$$
\begin{aligned}
\frac{\mathrm{d}}{\mathrm{d} r}\left(\rho_{\text {gas }}(r) \sigma_{r}^{2}\right) & +\frac{2 \rho_{\text {gas }}(r)}{r}\left(\sigma_{r}^{2}-\sigma_{\theta, \omega}^{2}\right)= \\
& =-\rho_{\text {gas }}(r) \cdot \frac{\mathrm{d} \Psi(r)}{\mathrm{d} r}
\end{aligned}
$$

where $\Psi$ is the gravitational potential of the cluster, $\sigma_{r}$ and $\sigma_{\theta, \omega}$ are the mass-weighted velocity dispersions in the radial and tangential directions respectively, and $\rho$ is the gas-mass density. For an isotropic system, it is

$$
\sigma_{r}=\sigma_{\theta, \omega}
$$

the pressure profile can be related to these quantities by

$$
P(r)=\sigma_{r}^{2} \cdot \rho_{\text {gas }}(r) .
$$

Substituting Eqs. (39) - (40) into Eq. (38), we have, for an isotropic sphere,

$$
\frac{\mathrm{d} P(r)}{\mathrm{d} r}=-\rho_{g a s}(r) \frac{\mathrm{d} \Psi(r)}{\mathrm{d} r} .
$$

For a gas sphere with temperature profile $T(r)$, the velocity dispersion becomes

$$
\sigma_{r}^{2}=\frac{k T(r)}{\mu m_{p}}
$$

where $k$ is the Boltzmann constant, $\mu \approx 0.609$ is the mean mass particle and $m_{p}$ is the proton mass. Substituting Eqs. (40) - (42) into Eq. (41), we obtain

$$
\frac{\mathrm{d}}{\mathrm{d} r}\left(\frac{k T(r)}{\mu m_{p}} \rho_{g a s}(r)\right)=-\rho_{g a s}(r) \frac{\mathrm{d} \Psi(r)}{\mathrm{d} r},
$$

or, equivalently,

$$
-\frac{\mathrm{d} \Psi(r)}{\mathrm{d} r}=\frac{k T(r)}{\mu m_{p} r}\left[\frac{\mathrm{d} \ln \rho_{g a s}(r)}{\mathrm{d} \ln r}+\frac{\mathrm{d} \ln T(r)}{\mathrm{d} \ln r}\right] .
$$

Now the total gravitational potential of the cluster is:

$$
\Psi(r)=\Psi_{N}(r)+\Psi_{C}(r) .
$$

It is worth underlining that if we consider only the standard Newtonian potential and its derivative in Eq. (43), the total cluster mass $M_{c l, N}(r)$ (the standard estimation of clusters mass in a CDM scenario) is composed by gas mass + galaxies mass + dark matter and it is given by the expression:

$$
\begin{aligned}
M_{c l, N}(r) & =M_{g a s}(r)+M_{g a l}(r)+M_{D M}(r)= \\
& =-\frac{k T(r)}{\mu m_{p} G} r\left[\frac{\mathrm{d} \ln \rho_{g a s}(r)}{\mathrm{d} \ln r}+\frac{\mathrm{d} \ln T(r)}{\mathrm{d} \ln r}\right]
\end{aligned}
$$

Generally the galaxy contribution is considered negligible with respect to the other two components so we have:

$$
\begin{aligned}
& M_{c l, N}(r) \approx M_{\text {gas }}(r)+M_{D M}(r) \approx \\
& \approx-\frac{k T(r)}{\mu m_{p}} r\left[\frac{\mathrm{d} \ln \rho_{g a s}(r)}{\mathrm{d} \ln r}+\frac{\mathrm{d} \ln T(r)}{\mathrm{d} \ln r}\right] .
\end{aligned}
$$

Since the gas-mass estimates are provided by X-ray observations, the equilibrium equation can be used to derive the amount of dark matter and to reconstruct its spatial distribution in a cluster of galaxies.

Inserting the previously defined extended-corrected potential of Eq. (44) into Eq. (43), we obtain:

$$
-\frac{\mathrm{d} \Psi_{N}}{\mathrm{~d} r}-\frac{\mathrm{d} \Psi_{C}}{\mathrm{~d} r}=\frac{k T(r)}{\mu m_{p} r}\left[\frac{\mathrm{d} \ln \rho_{g a s}(r)}{\mathrm{d} \ln r}+\frac{\mathrm{d} \ln T(r)}{\mathrm{d} \ln r}\right],
$$

from which the extended-corrected mass estimate follows:

$$
\begin{aligned}
M_{c l, E C}(r) & +\frac{r^{2}}{G} \frac{\mathrm{d} \Psi_{C}(r)}{\mathrm{d} r}= \\
& =-\frac{k T(r)}{\mu m_{p} G} r\left(\frac{\mathrm{d} \ln \rho_{g a s}(r)}{\mathrm{d} \ln r}+\frac{\mathrm{d} \ln T(r)}{\mathrm{d} \ln r}\right) .
\end{aligned}
$$

Since the use of a corrected potential avoids, in principle, the additional requirement of dark matter, the total cluster mass, in this case, is given by only the baryonic matter counterparts:

$$
M_{c l, E C}(r)=M_{g a s}(r)+M_{g a l}(r),
$$

that can be entirely evaluated by observational data. The mass density in the $\Psi_{C}$ term is

$$
\rho_{c l, E C}(r)=\rho_{\text {gas }}(r)+\rho_{\text {gal }}(r),
$$

with the density components derived from observations. Considering that the right term in Eq. (47) is the total Newtonian mass estimation for a cluster of galaxies, we easily derive that the corrective term in the gravitational potential works in mimicking an effective dark matter contribution:

$$
\frac{r^{2}}{G} \frac{\mathrm{d} \Psi_{C}}{\mathrm{~d} r}(r)=M_{c l, N}(r)-M_{c l, E C}(r) .
$$

But in our approach, instead of requiring new kind of particles, it arises by the interaction of baryonic matter with the scalar field scalar field.

We have hence performed a best-fit analysis of the theoretical estimation of dark matter, Eq. (50)

$$
M_{d m, t h}(r ; \beta, L) \doteq M_{e f f}(r ; \beta, L)=\frac{r^{2}}{G} \frac{\mathrm{d} \Psi_{C}(r)}{\mathrm{d} r}(r)
$$


which depends on scalar field parameters through the potential $\Psi_{C}$, versus the same but observationally-derived quantity,

$$
M_{d m, o b s}(r)=M_{c l, N}(r)-M_{c l, E C}(r) .
$$

We underline here that we could not fit directly the observed baryonic mass because of the great difference in order of magnitude between $M_{c l, N}(r)$ and $M_{c l, E C}(r)$, working this last one like a small perturbation to the total mass estimation. It is clear that the term corresponding to our theoretical derived dark matter quantity is much bigger than the baryonic contribution, and even a small and acceptable deviation of only $1 \%$ in it would have been translated in a larger deviation of $10 \%$ in the baryonic one.

Since not all the data involved in the above estimations have measurable errors, we cannot perform an exact $\chi$ square minimization. Actually, we can minimize the quantity:

$\chi_{C l}^{2}\left(\left\{\theta_{i}\right\}\right)=\frac{1}{\mathcal{N}-n_{p}-1} \cdot \sum_{i=1}^{\mathcal{N}} \frac{\left(M_{d m, o b s}^{i}-M_{d m, t h}^{i}\left(\left\{\theta_{i}\right\}\right)^{2}\right.}{M_{d m, t h}^{i}\left(\left\{\theta_{i}\right\}\right)}$

where $\mathcal{N}$ is the number of data and $n_{p}=2$ is the free parameters number of the model and $\left\{\theta_{i}\right\}=\{\beta, L\}$. As usual we find the minimum in $\chi$-square running MCMCs. Even if the convergence is achieved after few thousand steps of the chain, we have decided to run longer chains of $10^{5}$ points to reduce the noise from the histograms and avoid under- or over- estimations of errors on the parameters.

\section{Gas Density Model}

The gas density distribution of the clusters in the sample is described by the analytic model proposed in 90]. Such a model modifies the classical $\beta$-model to represent the characteristic properties of the observed X-ray surface brightness profiles, i.e. the power-law-type cusps of gas density in the cluster center, instead of a flat core and the steepening of the brightness profiles at large radii. Eventually, a second $\beta$-model, with a small core radius, is added to improve the model close to the cluster cores. The analytical form for the particle emission is given by:

$$
\begin{aligned}
n_{p} n_{e} & =n_{0}^{2} \cdot \frac{\left(r / r_{c}\right)^{-\alpha}}{\left(1+r^{2} / r_{c}^{2}\right)^{3 \beta-\alpha / 2}} \cdot \frac{1}{\left(1+r^{\gamma} / r_{s}^{\gamma}\right)^{\epsilon / \gamma}}+ \\
& +\frac{n_{02}^{2}}{\left(1+r^{2} / r_{c 2}^{2}\right)^{3 \beta_{2}}},
\end{aligned}
$$

which can be easily converted to a mass density using the relation:

$$
\rho_{\text {gas }}=n_{T} \cdot \mu m_{p}=\frac{1.4}{1.2} n_{e} m_{p},
$$

where $n_{T}$ is the total number density of particles in the gas. The resulting model has a large number of parame- ters, some of which do not have a direct physical interpretation. While this can often be inappropriate and computationally inconvenient, it suits well our case, where the main requirement is a detailed qualitative description of the cluster profiles.

In [90], Eq. (54) is applied to a restricted range of distance from the cluster center, i.e. between an inner cutoff $r_{\text {min }}$, chosen to exclude the central temperature bin $(\approx 10 \div 20 \mathrm{kpc})$ where the ICM is likely to be multi-phase, and $r_{\text {det }}$, where the X-ray surface brightness is at least $3 \sigma$ significant. We have extrapolated the above function to values outside this restricted range using the following criteria:

- for $r<r_{\min }$, we have performed a linear extrapolation of the first three terms out to $r=0 \mathrm{kpc}$;

- for $r>r_{\text {det }}$, we have performed a linear extrapolation of the last three terms out to a distance $\bar{r}$ for which $\rho_{\text {gas }}(\bar{r})=\rho_{c}, \rho_{c}$ being the critical density of the Universe at the cluster redshift: $\rho_{c}=$ $\rho_{c, 0} \cdot(1+z)^{3}$. For radii larger than $\bar{r}$, the gas density is assumed constant at $\rho_{\text {gas }}(\bar{r})$.

We point out that, in Table凹 the radius limit $r_{\min }$ is almost the same as given in the previous definition. When the value given by [90] is less than the cD-galaxy radius, which is defined in the next section, we choose this last one as the lower limit. On the contrary, $r_{\max }$ is quite different from $r_{d e t}$ : it is fixed by considering the higher value of temperature profile and not by imaging methods.

We then compute the gas mass $M_{\text {gas }}(r)$ and the total mass $M_{c l, N}(r)$, respectively, for all clusters in our sample, substituting Eq. (54) into Eqs. (55) and (45), respectively; the gas temperature profile is described in details in $\S$ ЩI 2. The resulting mass values, estimated at $r=r_{\max }$, are listed in Table

\section{Temperature Profiles}

As stressed in $\S$ IIB 1 for the purpose of this work, we need an accurate qualitative description of the radial behavior of the gas properties. Standard isothermal or polytropic models, or even the more complex one proposed in [90], do not provide a good description of the data at all radii and for all clusters in the present sample. We hence describe the gas temperature profiles using the straightforward X-ray spectral analysis results, without the introduction of any analytic model.

X-ray spectral values have been provided by A. Vikhlinin (private communication). A detailed description of the relative spectral analysis can be found in [89].

\section{Galaxy Distribution Model}

The galaxy density can be modelled as proposed by [2]. Even if the galaxy distribution is a point-distribution 
instead of a continuous function, assuming that galaxies are in equilibrium with gas, we can use a $\beta$-model, $\propto$ $r^{-3}$, for $r<R_{c}$ from the cluster center, and a steeper one, $\propto r^{-2.6}$, for $r>R_{c}$, where $R_{c}$ is the cluster core radius (its value is taken from [90]). Its final expression is:

$$
\rho_{\text {gal }}(r)= \begin{cases}\rho_{\text {gal }, 1} \cdot\left[1+\left(\frac{r}{R_{c}}\right)^{2}\right]^{-\frac{3}{2}} & r<R_{c} \\ \rho_{g a l, 2} \cdot\left[1+\left(\frac{r}{R_{c}}\right)^{2}\right]^{-\frac{2.6}{2}} & r>R_{c}\end{cases}
$$

where the constants $\rho_{\text {gal,1 }}$ and $\rho_{\text {gal,2 }}$ are chosen in the following way:

- 2] provides the central number density of galaxies in rich compact clusters for galaxies located within a $1.5 \mathrm{~h}^{-1} \mathrm{Mpc}$ radius from the cluster center and brighter than $m_{3}+2^{m}$ (where $m_{3}$ is the magnitude of the third brightest galaxy): $n_{\text {gal }, 0} \sim 10^{3} h^{3}$ galaxies $\mathrm{Mpc}^{-3}$. Then we fix $\rho_{\text {gal }, 1}$ in the range $\sim 10^{34} \div 10^{36} \mathrm{~kg} / \mathrm{kpc}^{3}$.

- the constant $\rho_{\text {gal,2 }}$ has been fixed with the only requirement that the galaxy density function has to be continuous at $R_{c}$.

For any cluster we assume that the galaxy population also consists in a cD galaxy, a giant elliptical galaxy with a diffuse envelope which is generally located at the center of clusters and whose typical mass is in the range $10^{12} \div$ $10^{13} M_{\odot}$. The cD galaxy density has been modeled as described in [75]; they use a Jaffe model of the form:

$$
\rho_{C D \text { gal }}=\frac{\rho_{0, J}}{\left(\frac{r}{r_{c}}\right)^{2}\left(1+\frac{r}{r_{c}}\right)^{2}},
$$

where $r_{c}$ is the core radius while the central density is obtained from $M_{J}=\frac{4}{3} \pi R_{c}^{3} \rho_{0, J}$. The mass of the cD galaxy has been fixed at $1.14 \times 10^{12} M_{\odot}$, with $r_{c}=R_{e} / 0.76$, with $R_{e}=25 \mathrm{kpc}$ being the effective radius of the galaxy. The central galaxy for each cluster in the sample is assumed to have approximately this stellar mass.

We have tested the effect of varying galaxy density with the central density parameter $\rho_{\text {gal }, 1}$ in the above range $\sim 10^{34} \div 10^{36} \mathrm{~kg} / \mathrm{kpc}^{3}$ on the cluster with the lowest mass, namely A262. In this case, we would expect greater variations with respect to other clusters; the result is that the contribution due to galaxies and cD-galaxy gives a variation $\leq 1 \%$ to the final estimate of fit parameters.

Finally, we have assumed that the total galaxycomponent mass (galaxies plus $\mathrm{cD}$-galaxy masses) is $\approx$ $20 \div 25 \%$ of the gas mass: in [74], the mean fraction of gas versus the total mass (with dark matter) for a cluster is estimated to be $15 \div 20 \%$, while the same quantity for galaxies is $3 \div 5 \%$. This means that the relative mean mass ratio gal-to-gas in a cluster is $\approx 20 \div 25 \%$. We have varied the parameters $\rho_{g a l, 1}, \rho_{g a l, 2}$ and $M_{J}$ in their previous defined ranges to obtain a mass ratio between total galaxy mass and total gas mass which lies in this range. At the end the cD-galaxy is dominant with respect to the other galaxies only in the inner region (below $100 \mathrm{kpc}$ ). As already stated in $\S$ IIIB 1. cluster innermost regions have been excluded from our analysis and so the contribution due to the cD-galaxy is practically negligible in our analysis. The gas is, as a consequence, the dominant visible component, starting from innermost regions out to large radii, being galaxy mass only $20 \div 25 \%$ of gas mass.

\section{Uncertainties on mass profiles}

Uncertainties on the cluster total mass profiles have been estimated performing Monte-Carlo simulations [63]. We proceed to simulate temperature profiles and choose random radius-temperature values couples for each bin which we have in our temperature data given by [89]. Random temperature values have been extracted from a Gaussian distribution centered on the spectral values, and with a dispersion fixed to its $68 \%$ confidence level. For the radius, we choose a random value inside each bin. We have performed 2000 simulations for each cluster and perform two cuts on the simulated profile. First, we exclude those profiles that give an unphysical negative estimate of the mass: this is possible when our simulated couples of quantities give rise to too high temperaturegradient. After this cut, we have $\approx 1500$ simulations for any cluster. Then we have ordered the resulting mass values for increasing radius values. Extreme mass estimates (outside the $10 \div 90 \%$ range) are excluded from the obtained distribution, in order to avoid other high mass gradients which give rise to masses too different from real data. The resulting limits provide the errors on the total mass. Uncertainties on the electron-density profiles have not been included in the simulations, being them negligible with respect to those of the gas-temperature profiles.

\section{Low surface brightness galaxies}

For the analysis of galactic scales we have used a sample of the so-called low surface brightness (LSB) galaxies and dwarf galaxies. It is still unclear to what extent rotation curves in bright spiral galaxies may give clues about the profile of both visual and dark matter, mainly because they are poor in gas content, so that rotation curves can be hardly detected out to sufficiently large radii where they are supposed to be dark matter dominated. Moreover they also show some typical complex features, such as extended spiral arms or barred structure that can lead to consistent non-circular motions and thus making difficult the interpretation of data. On the other side, LSB galaxies are supposed to be dark matter dominated at all radii, and therefore the analysis of their rotation curves can yield important clues about it. 
Effectively, LSB galaxies exhibit a large discrepancy between the detectable and the Newtonian dynamics mass within the optical disk [12, 30, 54, 80]. They are also a little challenge in the framework of CDM model because predictions from CDM based simulations have revealed disagreement with observational profiles of several dwarf galaxies [55]. In particular, data indicate much less cuspy distributions of matter than the simulations, and possible solutions such as feedback effect due to star formation have been excluded [86] by the observed low star formation ratio, at present and in the past, from which it can be deduced that star formation rate has never been important enough in these galaxies to modify their structure. Then, alternative solutions to Newtonian dynamics are also possible.

The chosen galaxies for our analysis come from a sample of 15 elements with high resolution $\mathrm{H} \alpha / \mathrm{HI}$ rotation curves extracted from the larger sample described in [31]. This sample was selected by [18] using the criteria of the contemporary availability of data on rotation curves, on surface photometry in the $\mathrm{R}$ band and on surface gasmass density. Rotation curves were derived from spectrographic observations performed by the authors themselves of 31], while the photometry of the stellar disk and $\mathrm{H} \alpha / \mathrm{HI}$ surface densities are collected from literature. For a more complete and detailed discussion one can mainly refers to 79 .

The final sample does not constitute a complete sample of dwarf and LSB galaxies, but contains galaxies in a wide range in luminosity and surface brightness for which high-quality rotation curves are available. Therefore it is well suited for testing scalar field mechanism in late-type dwarf and LSB galaxies.

Moving to the way of modeling our spiral galaxies, we have to specify the properties of stellar and gas distribution. Stars are generally supposed being distributed in a thin and circularly symmetric disk, with the surface density $\Sigma(R)$ derived from the observed surface brightness distribution through the relation:

$$
\Sigma(R)=10^{-0.4\left(\mu(R)-\mu_{R, \odot}-C\right)},
$$

where $\mu_{R, \odot}=4.46$ is the solar magnitude in the $\mathrm{R}$ band and $C=21.572$ is the needed constant for converting surface brightness from magnitude units, mag/ $\operatorname{arcsec}^{2}$, to linear units, $L_{\odot} / \mathrm{pc}^{2}$. The luminosity surface density is commonly fitted with the exponential disk model [41]:

$$
\Sigma(R)=\Sigma_{0} \exp \left(-R / R_{d}\right),
$$

where $\Sigma_{0}$ is the central surface brightness and $R_{d}$ is the disk scale length. Of course, in determining our theoretical rotation curve, we need the stellar mass density, that can be obtained from the luminosity one by simply multiplying it for the stellar mass-to-luminosity ratio, $Y_{*}$, which is, together with our scalar field parameters, the third free parameter having to be constrained with the fitting procedure.

Modeling the gas density is more complicated because we do not have an analytical function able to describe its behavior at all radii and because the profile is very disturbed. Using [79] plots and images, we fit the outer radii profile with a linear relation (in magnitude unities), while the inner one is generally fitted by simply interpolating data, with any analytical expression (generally a polynomial one) which well fits data points. We then check if the model works well by comparing the obtained total gas mass with the same quantity but evaluated by extrapolation from observational data. We verified that we only need very small normalization constants, in the range $(0.95,1.05)$, to fully match data values. Finally, we multiply the gas density with the factor 1.4 for including helium contribution.

With these model components, the general expression for the rotation velocity

$$
v_{c}^{2}(R)=\left.R \frac{\mathrm{d} \Psi(R, z)}{\mathrm{d} R}\right|_{z=0},
$$

can be decomposed in the following terms:

$$
v_{c}^{2}(R)=v_{c, N}^{2}(R)+v_{c, C}^{2}(R),
$$

with

$$
v_{c, N}^{2}(R)=\left.R \frac{\mathrm{d} \Psi_{N}(R, z)}{\mathrm{d} R}\right|_{z=0}
$$

and

$$
v_{c, C}^{2}(R)=\left.R \frac{\mathrm{d} \Psi_{C}(R, z)}{\mathrm{d} R}\right|_{z=0},
$$

the Newtonian and the corrective contributions to the rotational velocity from the respective terms in the total gravitational potential. Anyone of the previous terms can then be decomposed in two different component elements, concerning the two mass components, namely stars and gas. For stars we have:

$$
v_{c, N}^{\text {star } 2}\left(R ; Y_{*}\right)=\left.R \frac{\mathrm{d} \Psi_{N}^{s t a r}\left(R, z ; Y_{*}\right)}{\mathrm{d} R}\right|_{z=0},
$$

where we make explicit that this term depends on the free parameter $Y_{*}$ which appears in the stellar density inside the potential expression. When this term is not present in the available data, we use the expression given in [6] for the case of an exponential disk and with $Y_{*}=1$, i.e.

$$
\begin{aligned}
& v_{c, N}^{s t a r} 2(R)=4 \pi \mathrm{G} \Sigma_{0} R_{d}(y)^{2} . \\
& \text { - }\left[I_{0}(y) K_{0}(y)-I_{1}(y) K_{1}(y)\right] \text {, }
\end{aligned}
$$

with $y=R / 2 R_{d}$. For gas we have

$$
v_{c, N}^{\text {gas } 2}(R)=\left.R \frac{\mathrm{d} \Psi_{N}^{\text {gas }}(R, z)}{\mathrm{d} R}\right|_{z=0},
$$

and, as in the stellar case, when this kind of data is not available, we derive it in a numerical way by using the 
modeled gas density. From the corrective term to the potential we have

$$
v_{c, C}^{\operatorname{star} 2}\left(R ; Y_{*}, \beta, L\right)=\left.R \frac{\mathrm{d} \Psi_{C}^{\text {star }}\left(R, z ; Y_{*}, \beta, L\right)}{\mathrm{d} R}\right|_{z=0}
$$

and

$$
v_{c, C}^{\text {gas } 2}(R ; \beta, L)=\left.R \frac{\mathrm{d} \Psi_{C}^{\text {gas }}(R, z ; \beta, L)}{\mathrm{d} R}\right|_{z=0} ;
$$

both these two quantities are derived numerically. Finally, the total rotation velocity is the sum in quadrature of all these elements, i.e.

$$
\begin{aligned}
v_{c}^{2}(R) & =v_{c, N}^{\text {star } 2}\left(R ; Y_{*}\right)+v_{c, C}^{\text {star } 2}\left(R ; Y_{*}, \beta, L\right)+ \\
& +v_{c, N}^{\text {gas } 2}(R)+v_{c, C}^{\text {gas } 2}(R ; \beta, L)
\end{aligned}
$$

and the chi-square function is:

$$
\chi_{\mathrm{LSB}}^{2}\left(\left\{\theta_{i}\right\}\right)=\sum_{j=1}^{\mathcal{N}} \frac{\left(v_{c, t h}\left(R_{i},\left\{\theta_{i}\right)\right\}-v_{c, o b s}\left(R_{i}\right)\right)^{2}}{\sigma_{i}^{2}}
$$

where $\mathcal{N}$ is the number of data, $\sigma_{i}^{2}$ are the measurement variances and $\left\{\theta_{i}\right\}=\left\{\beta, L, Y_{*}\right\}$ is the parameters theory vector. Even in this case, like for supernovae and clusters, we use MCMC method for minimizing the chisquare function and deriving errors on the fit parameters.

\section{RESULTS AND DISCUSSION}

In this section we are going to discuss the obtained results with the goal to achieve a comprehensive background where scalar field works at various scales. In particular, we are going to search for possible trends and correlations between observable quantities and theoretical scalar field parameters in the various class of the considered astrophysical objects. SNeIa will be discussed alone because of the difficulties in interpreting their results in this general context. On the other hand, clusters and galaxies seem to be tied in a common picture by a rescaling process.

\section{A. Supernovae: results}

Difficulties come from the SNeIa analysis. In this case we have performed MCMC analysis leaving the scalar field parameters free, with the only minimal requirement that $L>0$, being it a length, and that $\beta>0$, because of only the term $\beta^{2}$ appears to be involved in the Eq. (31) and no possibility to discriminate between positive and negative values is given.

The parameter $\lambda$ may be considered as a length related to stellar scale, to typical supernovae explosion, and/or to stellar formation. Since its exact value is unknown, we have varied it on a grid ranging from $\lambda=10^{-3} \mathrm{~h}^{-1}$ as the minimum, and corresponding to a length of $\approx 1 \mathrm{kpc}$ (assuming $H_{0}=74.2$ as in [50]), up to $\lambda=1 \mathrm{~h}^{-1}$ as the maximum, and corresponding to a length $\approx 1 \mathrm{Mpc}$.

In Table 1 we have also reproduced the values of chisquare function evaluated at the best fit points: they are completely equal to the same quantity evaluated for a CPL model with $\Omega_{m, 0}, w_{0}$ and $w_{a}$ as fit parameters and a gravitational constant fixed at its usual Newtonian value (constant in time and scale). For CPL model we have obtained the following results:

$$
\begin{aligned}
\Omega_{m, 0} & =0.273_{-0.133}^{+0.091} \\
w_{0} & =-0.954_{-0.275}^{+0.210} \\
w_{a} & =0.003_{-0.150}^{+0.201} \\
\chi_{\text {best }} & =465.665
\end{aligned}
$$

If we want to compare the reliability of our model against the CPL one, we can use a Bayesian type test as BIC. It is defined as $\mathrm{BIC}=-2 \ln \mathcal{L}+k \ln N$ [76], with $-2 \ln \mathcal{L}$ being the chi-square value and $\mathcal{L}$ the likelihood function, $k$ the number of parameters of the model and $N$ the number of points in the dataset. The best model has the lowest BIC; in particular, when comparing two different models, if the difference between the two $\mathrm{BIC}$ values is $\triangle \mathrm{BIC}<2$, than there is no significant difference between the models; if $2<\Delta \mathrm{BIC}<5$, this difference is substantial; if $5<\Delta \mathrm{BIC}<10$ there is a "strong" evidence in favor of the model with the lowest $\mathrm{BIC}$ value; while for $\Delta \mathrm{BIC}>10$ this evidence is "decisive" (following the most used value scale, the "Jeffreys' scale", defined and reported in [47]).

If we consider that in the CPL case we have three fit parameters as in our scalar field model, we can easily deduce that there are no significative differences between the CPL and the scalar field model.

Things change slightly if we consider a $\Lambda$ CDM model with a Newtonian gravitational constant and only one free parameter, $\Omega_{m, 0}$. In this case, even if the chi-square has the same value of our scalar field model, we have only one parameter, so that $\Lambda$ CDM model with a Newtonian gravitational constant is strongly favored with respect to a scalar field model with a varying gravitational constant (we may underline that this is an obvious consequence of the BIC definition, where the $k \ln N$ tends to support models with a smaller number of parameters). We will return later on the chi-square values for a further discussion.

Now we can take a look to the best fit values of model parameters. For what concerning the matter content parameter, we can observe that for $\Omega_{m, 0}$ they are slightly higher than the latest estimation 50 ], $\Omega_{m, 0} \approx 0.24$, from combining WMAP results with BAO and and the Hubble constant measurements, but they are aligned with usual values derived from only-SN analysis, which generally lead to higher values for this parameter [15].

We have also to stress that such a high value for $\Omega_{m, 0}$, although comparable with the usual contribution generally attributed to dark matter, is not contradictory at 
Table I. Supernovae. Column 1: $\lambda$ value. Column 2: chi square value evaluated at the best fit values for fitting parameters. Column 3: present matter content, $\Omega_{m, 0}$. Column 4: coupling parameter $\beta$ from scalar field ( $\sigma$ confidence interval). Column 5: gravitational length $L$ from scalar field ( $1 \sigma$ confidence interval).

\begin{tabular}{ccccc}
\hline$\lambda$ & $\chi_{\text {best }}^{2}$ & $\Omega_{m, 0}$ & $\beta$ & $\begin{array}{c}L \\
\left(h^{-1}\right)\end{array}$ \\
& & & & $(\mathrm{Mpc})$ \\
\hline $10^{-3}(\approx 1 k p c)$ & 465.610 & $0.292_{-0.023}^{+0.023}$ & $0.135_{-0.099}^{+0.459}$ & $0.145_{-0.110}^{+0.677}$ \\
$10^{-2}(\approx 10 k p c)$ & 465.659 & $0.295_{-0.024}^{+0.027}$ & $0.120_{-0.088}^{+0.326}$ & $0.158_{-0.119}^{+0.743}$ \\
$10^{-1}(\approx 100 k p c)$ & 465.633 & $0.301_{-0.026}^{+0.040}$ & $0.114_{-0.083}^{+0.305}$ & $0.174_{-0.136}^{+0.758}$ \\
$10^{0}(\approx 1 M p c)$ & 465.707 & $0.298_{-0.024}^{+0.031}$ & $0.129_{-0.094}^{+0.347}$ & $0.159_{-0.123}^{+0.991}$ \\
\hline
\end{tabular}

all with our intent of explaining dark matter on smaller than cosmological scales and dark energy on cosmological scales with the same source, namely, the scalar field effective scalar field.

In fact we can easily consider that there are two elements contributing to the $\Omega_{m, 0}$ value: one coming from ordinary baryonic matter, $\Omega_{b, 0} \approx 0.04$, and one coming from the scalar field, thus acting as a dark matter-type component and scaling as $(1+z)^{3}$. As the discussion in next pages will show, this is a feasible possibility. Then, we have a contribution from scalar field to the acceleration of universe through an effective dark energy component, for an amount equal to the usually derived one $\left(\Omega_{c h, 0} \approx 0.70\right)$ and acting as a cosmological constant.

Discussions about the scalar field parameters are more tricky: they do not show significative statistical changes while varying $\lambda$. By applying Eq. (30) to the Constitution data set we are implicitly assuming that the $\lambda$ length is the same for all supernovae; if we assume that these objects constitute an homogeneous astrophysical family, our hypothesis looks general and quite good.

The parameter $\beta$ quantifies the coupling of the scalar field with ordinary matter and mainly measures how much the effective gravitational constant deviates from the usual Newtonian one. From the cosmological analysis it is in the interval $[0.114,0.135]$. On the other side, the length L may be considered in this case as the "minimal" length for which the variation of the gravitational constant has "cosmological" effects ( i.e. detectable with the Hubble SNeIa diagram) and with the scalar-field mimicking a dark energy-type component. Our analysis shows that such a scale is $\sim 100 \mathrm{kpc}$.

Anyway, we have to combine all these results with the evidence that chi-square values are completely equal for any $\lambda$, then we have also to consider the possibility that our analysis is not well-based. In the top panel of Fig. (2) we have plotted how much the modified distance modulus in Eq. (30) differs from the usual expression,

$$
\mu(z)=5 \log \left((1+z) \int_{0}^{z} \frac{\mathrm{d} z}{h(z)}\right)+\mu_{0}
$$

If we consider that the modified distance modulus can be written as:

$$
\mu(z ; \beta, L ; \lambda)=\mu(z)+\frac{15}{4} \log \frac{G_{e f f}(z ; \beta, L ; \lambda)}{G_{e f f}(0 ; \beta, L ; \lambda)}
$$

the correction coming from the effective scalar field gravitational constant is negligible when compared to the usual distance modulus expression, being at most $\approx$ $0.08 \%$. Facing with this question, we have two possibilities:

1. our results do not come from an effective test of a scalar field mechanism, but are got stuck by the impossibility to detect changes in the gravitational constant, being the distance modulus mainly driven by the $\Omega_{m, 0}$ parameter in Eq. (72);

2. we are effectively testing a scalar field mechanism, but then we have the problem of including the obtained values for scalar field length $L$ in a consistent theoretical background.

As we can see in the middle and bottom panels of Fig. (2), we have different scenarios depending on the value of $\lambda$ :

- for $\lambda=10^{-3}$ we have an effective gravitational constant which is practically constant, exhibiting a change of only $\sim 0.003 \%$ in the redshift range $[0.005,1]$ and assessing at a value which differs from the Newtonian gravitational constant value for $\sim 3.6 \%$. In this case the relative contribution of the corrective term with respect of the usual one is $\approx 10^{-5 \%}$;

- for $\lambda=10^{-2}$, we have again a practically constant effective gravitational constant, with a change of $\sim 0.7 \%$ in the SNeIa redshift range, and assessing at a value which differs from the Newtonian gravitational constant value for $\sim 2.85 \%$. In this case the relative contribution of the corrective term with respect of the usual one is really small, $\approx 0.0006 \%$;

- for $\lambda=10^{-1}$ we have a more sensible rise of effective gravitational constant in the supernovae redshift range, reaching an asymptotic value bigger 

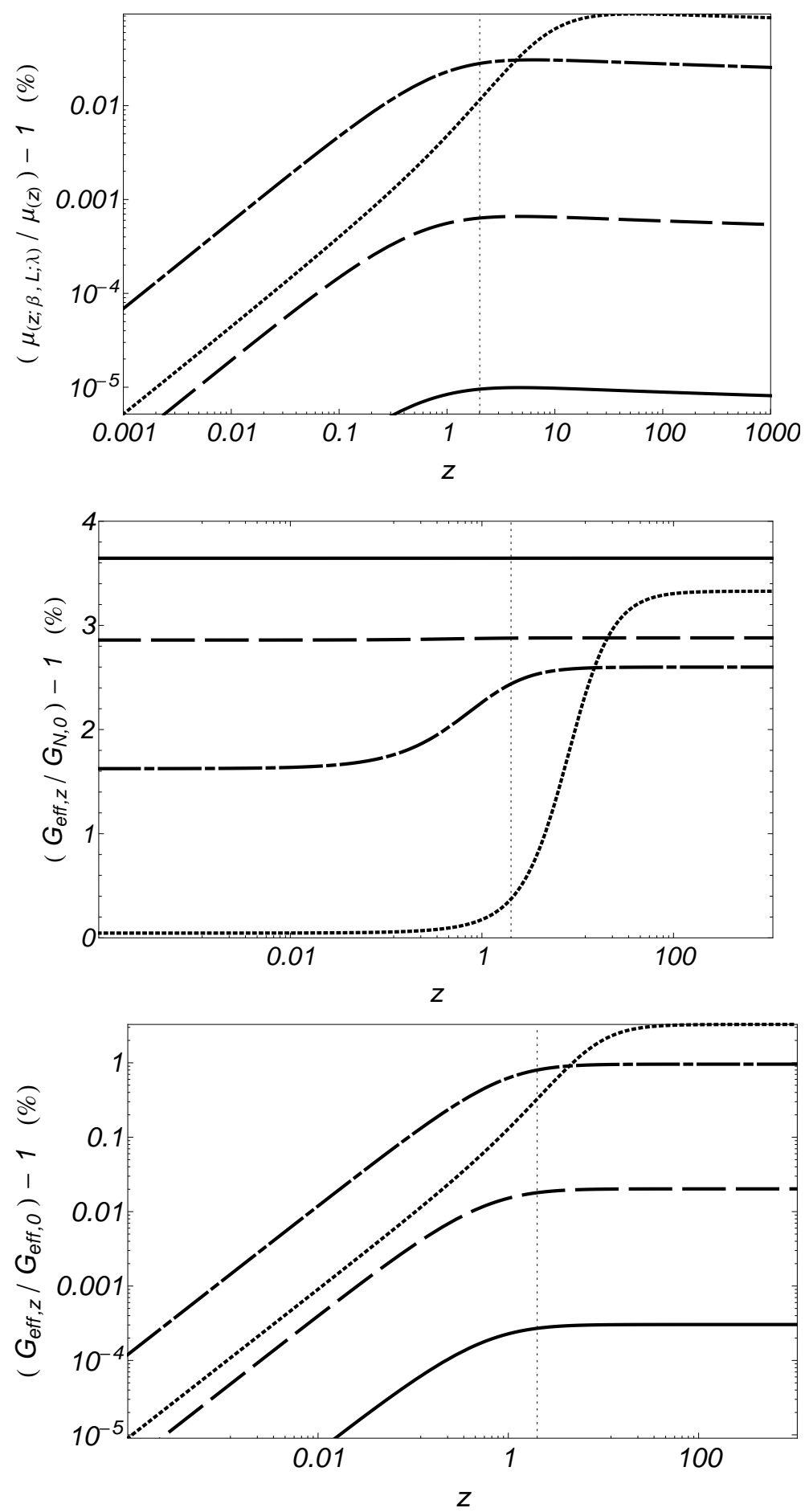

Figure 2. Continuous line is for $\lambda=10^{-3}(\beta=0.135 ; L=0.145 \mathrm{Mpc})$; dashed line is for $\lambda=10^{-2}(\beta=0.120 ; L=0.158$ $\mathrm{Mpc})$; dot-dashed line is for $\lambda=10^{-1}(\beta=0.114 ; L=0.174 \mathrm{Mpc})$; dotted line is for $\lambda=10^{0}(\beta=0.129 ; L=0.159 \mathrm{Mpc})$. The vertical dotted line shows the maximum redshift from SNeIa sample. Top panel. Deviations of the corrected version of distance modulus from the usual one. Middle panel. Ratio between the effective gravitational constant, Eq. (9), and the Newton gravitational constant value. This ratio quantifies the temporal evolution of the deviation of scalar field mechanism from Newtonian gravity. Bottom panel. Ratio between the effective gravitational constant, Eq. (9), and the present value of the same quantity. This ratio quantifies the temporal evolution of the effective gravitational constant. 
than Newtonian one for $\sim 2.6 \%$ at $z>50$. Following the large change in the redsfhift dependence of the effective gravitational constant, which can vary for $1 \%$ even in the supernovae redshift interval, the ratio between the corrective and the usual expression of distance modulus in this case is $\approx 0.028 \%$;

- for $\lambda=1$ the rising is less pronounced in the supernovae redshift range with respect of previous case, but goes over at $z \approx 15$ and reaches its asymptotic value at $z \sim 500$, being it $\sim 3.32 \%$ bigger than Newtonian gravitational constant. In this case the corrective distance modulus term reaches a maximum deviation of $\approx 0.08 \%$ around $z \sim 50$; in the same range the effective gravitational constant can vary for $\approx 3 \%$.

\section{B. Clusters of galaxies: results}

When considering clusters of galaxies we remind that in this case we left free the scalar field parameters, $\beta$ and $L$, with only the minimum requirement of their positiveness.

As it is possible to see by simple visual inspection, the only bad fit corresponds to the cluster RXJ1159: using the modelled matter densities described in $\S$ (IIIB 1) (IIIB 3), we obtain a too fast decreasing mass profile in the inner region reaching unphysical negative values. For this reason we will not consider it anymore in our considerations.

On the contrary, for all the other clusters we have good results, with mass estimations corresponding in the $1 \sigma$ confidence level. Errors contours reported in Fig. (3) (44) have two contributions: the main one comes from the statistically derived errors on mass observations, as described in $\S$ (IIIB4 and which produce the larger and irregular borders of the $1 \sigma$ confidence level; the smallest one comes from errors on fitting parameters.

What can be first noted is that for lower scales there is a large deviation between our theoretical estimation and the observed one. Typically one can locate this breakpoint in the range $[100,150] \mathrm{kpc}$. This is not a new thing when describing clusters of galaxies with modified gravities and it is not an intrinsic failure of our theoretical model. Similar issues are present in [21], where $f(R)$ gravity models are applied to clusters of galaxies. The same situation is in [13]: they use the the Metric - Skew - Tensor - Gravity (MSTG) as a generalization of the Einstein General Relativity and derive the gas mass profile of a sample of clusters with gas being the only baryonic component of the clusters. They consider some clusters included in our sample (in particular, A133, A262, A478, A1413, A1795, A2029, MKW4) and the same failing trend is found for $r \leq 200 \mathrm{kpc}$ : they overestimate gas mass in the inner regions with respect of the expected estimation from X-ray observations. In the same work there is also an interesting note about MOND theory ap- plied to clusters of galaxies: even if it is not possible to assess it really fails, surely we can see that MOND in clusters does not solve the dark matter problem because it again requires including a mass contribution other than the observed one.

The reason for this different behavior in the inner regions is in the break of the hypothesis of hydrostatic equilibrium. If the hypothesis of hydrostatic equilibrium is not correct, then we are in a regime where the fundamental relations Eqs. (38) - 431) are not working. As discussed in [89], the central - $70 \mathrm{kpc}$ - region of most clusters is strongly affected by radiative cooling and thus its physical properties cannot directly be related to the depth of the cluster potential well. This means that, in this region, the gas is not in hydrostatic equilibrium but in a multi-phase state. In this case, the gas temperature cannot be used as a good standard tracer. Among the main phenomena which causes this we have cooling flows, merger effects and asymmetric shapes. In particular, cooling flows produce a decrease in the temperature profile and consequently local higher gas densities which cannot be related directly to gravitational effects.

A coherent behavior is shown in our plots, Fig. (3) (41). We remind that there the distribution of dark matter is represented; higher densities from cooling flows produce higher non-gravitational-produced gas mass profiles, which result in a decrease of the dark matter one. In our case, we have that our theoretical dark matter profile (i.e. the effective dark matter mimicked by the different coupling of scalar field with baryonic mass) is higher than the observationally derived one (see for example Abell 262, Abell 383, Abell 478, Abell 1413, Abell 1991, Abell 2029; while for Abell A133 and Abell 1795 one can perceive the same trend but unfortunately the data do not extend too small enough radii).

However, a more detailed modelling for inner regions is out the purpose of this work, while we are here interested to show that the scalar field mechanism can be a valid alternative to dark matter in order to explain cluster dynamics. In this sense it is very illuminating that on the most part of objects in our sample there is a very good agreement between the scalar field model and the observationally derived dark matter profiles on a wide range of distances from the centers for any cluster, approximately in the interval $[100 ; 1000] \mathrm{kpc}$, or at least up to the maximum radii coming from observations.

Giving a more detailed glance to the absolute values of scalar field parameters for clusters, we can see that the coupling constant $\beta$ looks very well constrained in a really narrow range, $[1.888 ; 3.259]$; while the gravitational length $L$ can vary in the interval $[148.931 ; 1487.800] \mathrm{kpc}$, which seems consistent with the range depicted by other used characteristic clusters scale, as the virial radius in a $\Lambda$ CDM context.

To be more precise, if we consider (and exclude) three peculiar cases, these intervals can even be constrained in narrower windows: for $\beta$, the interval may be $[2.047 ; 2.786]$, while for $L$ it may be $[147.977 ; 1323.890]$. 

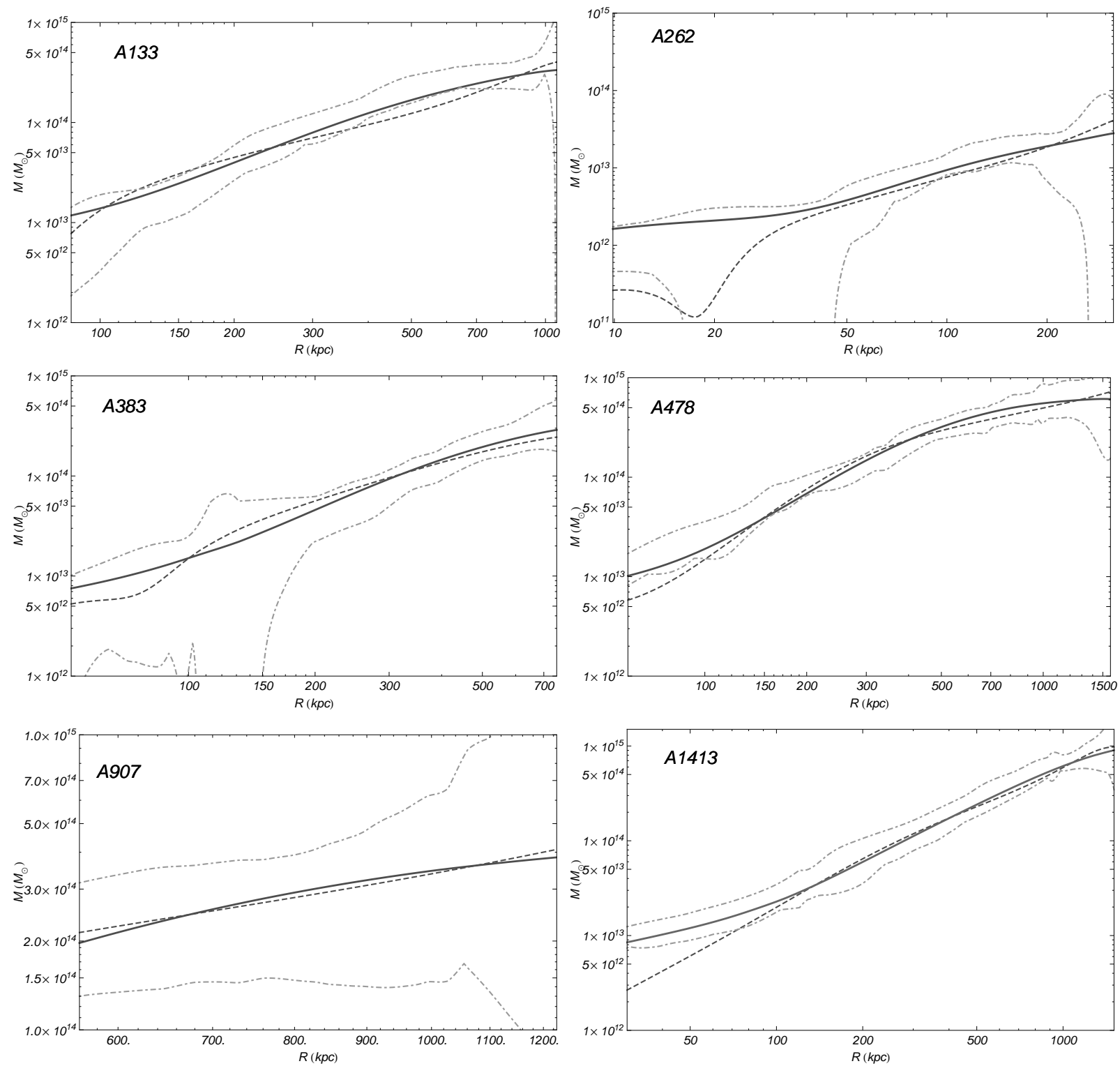

Figure 3. Dark matter profile vs radii for clusters of galaxies. Dashed line is the observationally derived estimation of dark matter, Eq. (52); solid line is the theoretical estimation for the effective dark matter component, Eq. (51); dot-dashed lines are the 1- $\sigma$ confidence levels given by errors on fitting parameters plus statistical errors on mass profiles as discussed in $\S$ ЩB 4

In [89], MKW4 and RXJ1159 are recognized as the strongest outliers in temperature profile, exhibiting very compact cooling regions and having a temperature peak smaller than other sample objects and located in inner region at $r \approx 50 \mathrm{kpc}$. RXJ1159 is better classified as an X-ray Over-Luminous Elliptical Galaxy; optically, this object appears as a nearly isolated elliptical galaxy but its X-ray luminosity and extent is typical of poor clusters. MKW4 is considered a group of galaxies or a poor cluster [64] too, so we have to consider them as different object from the rest of the sample. Moreover we cannot give any conclusion about this gravitational class (group of galaxies) because only one object has no statistical weight for any analysis.
Always in [89], the cluster Abell 2390 seems to elude typical clusters scaling relations; this is due to its unusual central cool region, which extends up to $r \sim 400 \mathrm{kpc}$, probably because the cold gas is pushed out from the center by radio lobes.

From these considerations, it is interesting to note that just these three objects result having the most different values for the coupling constant $\beta$ with respect of the other clusters and even seem to exhibit a peculiar trend. MKW4 (and RXJ1159) have more compact inner cool regions and this is associated with: 1. higher values for $\beta$, which means a larger coupling of scalar field with ordinary matter ( $\beta$ acts like a concentration parameter) and 2 . smaller value for the gravitational length. On the 

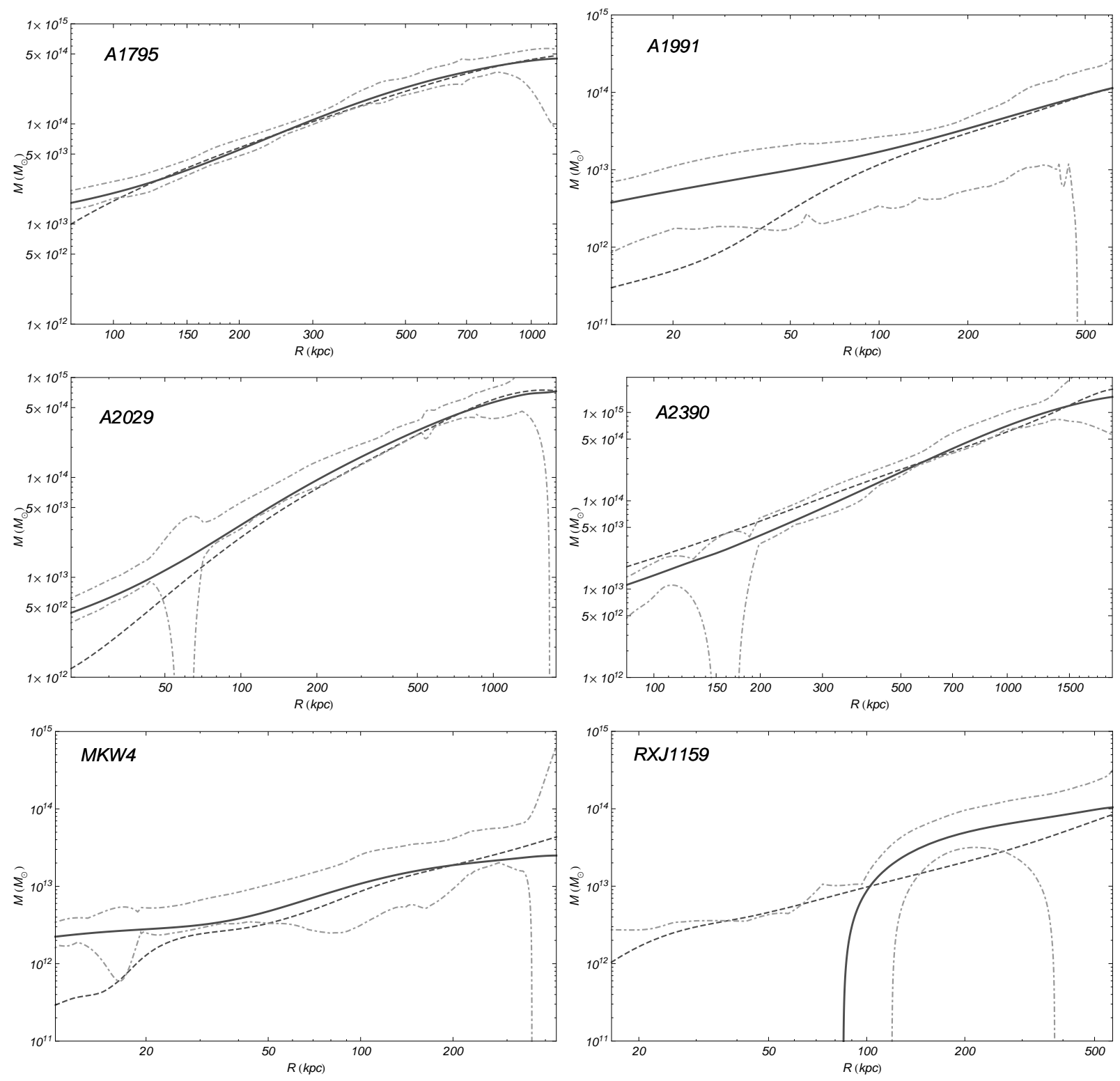

Figure 4. Same of Fig. (3).

contrary, Abell 2390 shows a larger cooling region, which corresponds to: 1 . smaller values for $\beta$ and 2 . larger for $L$.

We underline that in our approach it is very important to find these kind of correlations: if we want to make the scalar field mechanism the most general and basic possible, we need to find any possible link among its parameters and the physical properties of our analyzed gravitational systems, so being able to perform forecasts for other scaling properties and to recognize that a scalar field is acting even when it is not directly possible to derive easy quantities to be compared with data.

Going on in this search, we have plotted the scalar field length $L$ versus the virial radius of any cluster (bottom panel in Fig. (50), calculated using the relation [14, 38],

$$
r_{v i r}=1.95 \mathrm{~h}^{-1} \mathrm{Mpc}\left(\frac{<T>}{10 \mathrm{keV}}\right)^{1 / 2},
$$

where $h=0.742$ [50] and $\langle T\rangle$ is the average temperature for any cluster, derived from gas-weighted fit to the total cluster spectrum excluding the central region, and resulting more directly correlated to clusters mass than the X-ray emission weighted temperatures. Even if the two lengths result very different, with the scalar field parameter $L$ always smaller than the virial radius, we have a phenomenological relation between them (we have performed a weighted linear fit which results better than any higher order polynomial),

$$
\log L=3.0082 \cdot \log r_{v i r}-7.0476 \text {. }
$$


Table II. Clusters of galaxies. Column 1: Cluster name. Column 2: cluster total mass. Column 3: gas mass. Gas and total mass values are estimated at $r=r_{\max }$. Columns 4: gas mass weighted average temperature. Column 5: virial radius. Column 6: minimum observational radius. Column 7: maximum radius. Column 8: coupling parameter $\beta$ from scalar field ( $1 \sigma$ confidence interval). Columns 9: gravitational length $L$ from scalar field ( $1 \sigma$ confidence interval).

\begin{tabular}{|c|c|c|c|c|c|c|c|c|}
\hline name & $\begin{array}{c}M_{c l, N} \\
\left(10^{14} M_{\odot}\right)\end{array}$ & $\begin{array}{c}M_{\text {gas }} \\
\left(10^{13} M_{\odot}\right)\end{array}$ & $\begin{array}{l}<T> \\
(\mathrm{keV})\end{array}$ & $\begin{array}{c}r_{v i r} \\
(\mathrm{kpc})\end{array}$ & $\begin{array}{l}r_{\min } \\
(\mathrm{kpc})\end{array}$ & $\begin{array}{l}r_{\max } \\
(\mathrm{kpc})\end{array}$ & $\beta$ & $\begin{array}{c}L \\
(\mathrm{kpc})\end{array}$ \\
\hline A133 & .35874 & 2.73866 & 3.68 & 1694.26 & 86 & 1060 & $2.524_{-0.228}^{+0.259}$ & $888.557_{-2}^{+4}$ \\
\hline A262 & 0.445081 & 0.276659 & 1.92 & 1199.46 & 61 & 316 & $2.786_{-0.356}^{+0.397}$ & $147.977_{-}^{+}$ \\
\hline A383 & 2.79785 & 2.82467 & 4.36 & 1822.12 & 52 & 751 & $2.189_{-0.187}^{+0.206}$ & $728.246_{-}^{+}$ \\
\hline A 478 & 8.51 & 10. & 7.34 & 23 & 59 & 1580 & $2.106_{-0}^{+0}$ & $820.874_{-}^{+}$ \\
\hline A907 & $4.8^{\prime}$ & 6.3 & 5.44 & 39 & 563 & 1226 & $2.364_{-0}^{+0}$ & $594.207_{-}^{+}$ \\
\hline A1413 & 10.9598 & 9.32466 & 6.76 & 2259.35 & 57 & 1506 & $2.210_{-0}^{+0}$ & $1323.890_{-}^{+}$ \\
\hline A1795 & 5.44761 & 5.56245 & 5.52 & 2054.1 & 79 & 1151 & $2.224_{-0}^{+0}$ & $869.098_{-}^{+}$ \\
\hline A1991 & 1.24313 & 1.00530 & 2.23 & 1338.46 & 55 & 618 & $2.439_{-0}^{+0}$ & $534.918_{-}^{+}$ \\
\hline A 2029 & 8.92392 & 12.4129 & 7.59 & 2419.03 & 62 & 1771 & $2.047_{-0}^{+0}$ & $1073.050_{-}^{+}$ \\
\hline A 2390 & 20.9710 & 21.5 & 9.35 & 2481.14 & 83 & 1984 & $1.888_{-0.065}^{+0.067}$ & $1487.800_{-10}^{+90}$ \\
\hline MKW4 & 0.469503 & 0.283207 & 1.58 & 1068.31 & 60 & 434 & $3.259_{-0.737}^{+20.876}$ & $148.931_{-141.849}^{+621.309}$ \\
\hline RXJ1159 & 0.897997 & 0.433256 & 1.40 & 1115.81 & 64 & 568 & $3.412_{-0.722}^{+1.702}$ & $387.568_{-251.839}^{+601.661}$ \\
\hline
\end{tabular}
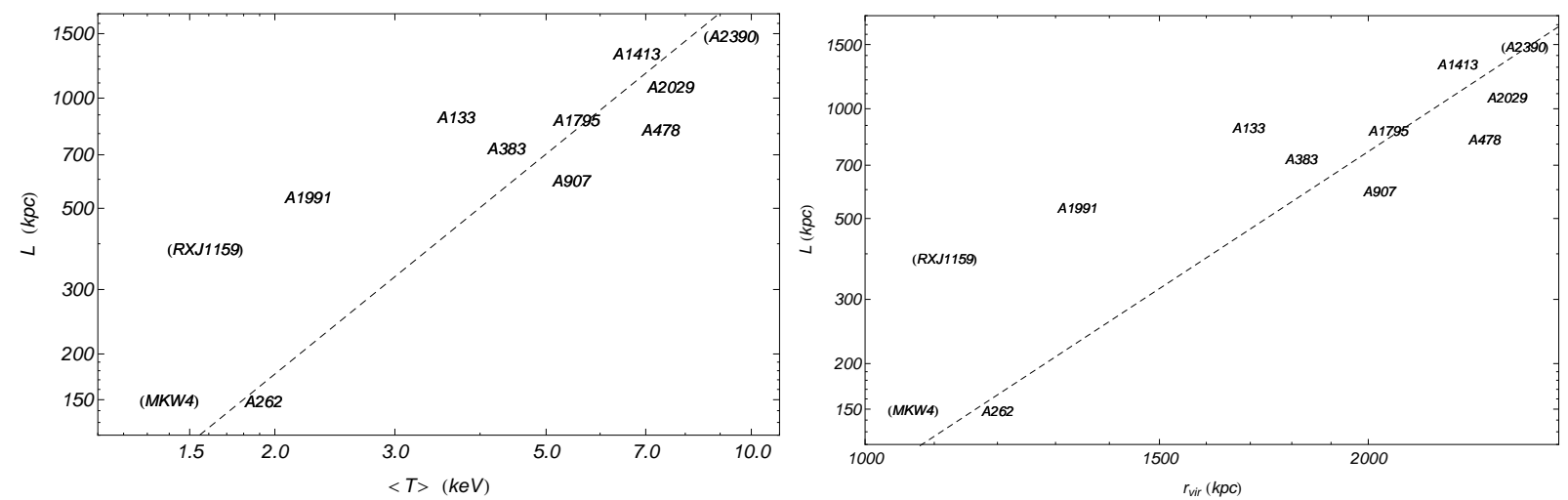

Figure 5. Scalar field length plotted versus mean (gas density weighted) cluster temperature (top panel) and the virial radius (bottom panel). Objects in brackets are excluded from fits as described in $\S$ (IVB).

From it one can see that the scalar field length scales as $L \propto r_{v i r}^{3} \sim M_{v i r}$, so that it may possible to find a relation between scalar field parameters and the virial (Navarro Frenk - White (NFW) model based) ones.

We have also checked a possible relation between $L$ and the mean temperature $\langle T\rangle$ (top panel in Fig. (5)), with the final result:

$$
\log L=1.51085 \cdot \log \langle T\rangle+1.79122 .
$$

This last results is again very intriguing, because for clusters of galaxies we have a mass-temperature relation [14],

$$
M_{\Delta} / T^{3 / 2} \propto H_{0} / H(z),
$$

where $\Delta$ is the overdensity level relative to the critical density at the cluster redshift, so that $M_{180}=M_{\text {vir }}$. Then we have to consider that for most of our clusters the redshift is very small so that the ratio $H_{0} / H(z)$ is almost equal to 1 . We remind that:

$$
L \propto<T>^{3 / 2} \text { and } L \propto r_{v i r}^{3} \sim M_{v i r},
$$

so we can deduce that our scalar field length follows perfectly the mass-temperature relation, being $L \propto<$ $T>^{3 / 2} \propto M_{v i r}$ and that any scatter in the previous relation can be attributed to differences in clusters redshifts.

Finally, we plotted in Fig. (6) the scaled temperature profiles versus the distance from the center of any clusters scaled with respect of the scalar field length $L$ obtained by fit. It is extremely interesting and not obvious 

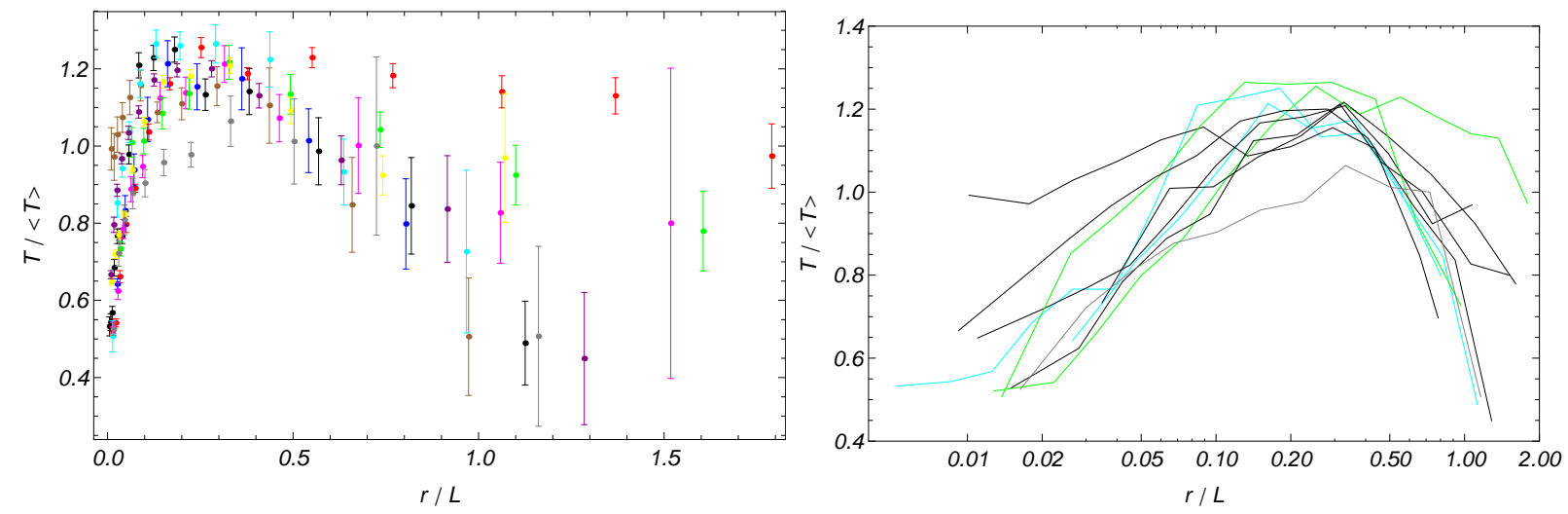

Figure 6. Temperature profiles for all clusters plotted as a function of distance from the center and in units of the scalar field length $L$. The temperatures are scaled to the mean (gas density weighted) cluster temperature. Only the two group galaxies, MKW4 and RXJ1159, have not been plotted. Colors in the bottom panel mean: black for clusters with $<T>$ greater than 5 Kev; cyan for $\langle T\rangle$ between 2.5 and $5 \mathrm{keV}$; green for $\langle T>$ less than $2.5 \mathrm{KeV}$; grey for Abell 2390 .

that the profiles are all re-scaled and self-similar as it happens in the usual approach. We can even say something more: in the scalar field approach we can see that some properties which are depicted in Fig. (16) of [89], as for example the different profiles among subgroups of clusters with mean temperature in different ranges (less than $2.5 \mathrm{keV}$, between 2.5 and $5 . \mathrm{keV}$ and greater than $5 \mathrm{keV}$ ) with distances rescaled with respect of $r_{500}$, here disappear. In fact, all the clusters form an homogeneous gravitational family (the only exception being Abell 2390, as discussed before). We could say that the scalar field length $L$ contains more fundamental information than the virial radius.

\section{LSB galaxies: results}

Taking into account LSB galaxies we have one parameter more than the clusters case: together with the intrinsic scalar field parameters we have the stellar massto-light ratio, $Y_{*}$, which we need for the conversion from the stellar surface photometry to the stellar mass density. In principle this parameter could be left free; but we have decided to put a prior on it derived from literature. In 86 they investigate star formation history and chemical evolution of LSB galaxies by modeling their observed spectro-photometric and chemical properties using a galactic chemical and photometric evolution model incorporating a detailed metallicity dependent set of stellar input data. Results show that $Y_{*}$ for this class of galaxies has usually values between $\sim 0.5$ and $\sim 2$. For this reason we have constrained the mass-to-light ratio to the interval $[0 ; 5]$ as a conservative hypothesis, and only in one case (UGC3851) we had to enlarge the interval up to 10 .

An important assumption about this parameter is that we assume it being constant over the whole range of data; this is an unavoidable and the most general assumption one can do without having details of stellar population distribution inside LSB galaxies.

A comment is in order for a correct evaluation of the results: during the analysis we have compared the theoretical rotation curves coming out from our scalar field model using only the observable-matter densities described in 79. with data firstly published in 31. These data, available in the SIMBAD data base, consist of the contributions to the total rotation curve coming from the stellar component (with an assumed mass-to-light ratio $Y_{*}=1$ ) and the gas one (with gas density normalized with a factor 1.4 for taking into account helium contribution), together with the total observed rotation curve (which can be considered an effect of dark matter in a CDM scenario or coming out from the interaction with a scalar field as in our approach). Both the total and the gas rotation curve have been submitted to a smoothing procedure for deleting irregularities mainly coming from two different elements. First, the assumption that has been made when deriving mass models from rotation curves is that there is symmetry, that all mass in on circular orbits and that there is continuity with radius. Raw data show scatter and non-circular motions which can produce virtual or ambiguous rotation velocities. Second, gas densities often show small-scale structures, irregularities and look clumpy at any distance from the center, and this can give possible large fluctuations in the rotation curve. In 31] the smoothing procedure is tested of course, and the smooth rotation curve is a very good approximation to the raw rotation curve. But some discrepancies with our theoretical estimations can be find because of we have used raw gas density profiles from [79].

If we consider that all gas contributions to rotation curve are multiplied to $\beta$ only, while the star ones to the combination $\beta \cdot Y_{*}$, in some cases, depending on the fit values and on their contribution, irregularities in the gas distribution are emphasized and may affect the total rotation curve profile. 
Table III. LSB galaxies. Column 1: UGC number. Column 2: Distance from the source literature. Column 3: disk central surface brightness in R-band, corrected for galactic extinction and inclination. Column 4: disk scale length. Column 5: total HI gas mass. Column 6: Maximum rotation velocity. Column 7: best fit stellar mass to light ratio ( $\sigma \sigma$ confidence interval). Column 8: coupling parameter $\beta$ from scalar field ( $1 \sigma$ confidence interval). Columns 9: gravitational length $L$ from scalar field $(1 \sigma$ confidence interval).

\begin{tabular}{ccccccccc}
\hline UGC & $\begin{array}{c}\mathrm{D} \\
(\mathrm{Mpc})\end{array}$ & $\begin{array}{c}\mu_{0, R} \\
\left(\mathrm{mag} / \mathrm{arcsec}^{-2}\right)\end{array}$ & $\begin{array}{c}R_{d} \\
(\mathrm{kpc})\end{array}$ & $\begin{array}{c}M_{H I} \\
\left(10^{8} M_{\odot}\right)\end{array}$ & $\begin{array}{c}V_{\max } \\
(\mathrm{km} / \mathrm{s})\end{array}$ & $\begin{array}{c}Y_{*} \\
\left(Y_{\odot}\right)\end{array}$ & $\beta$ & $\begin{array}{c}L \\
(\mathrm{kpc})\end{array}$ \\
\hline $\mathrm{U} 1230$ & 51 & 22.6 & 4.5 & 58.0 & 103 & $2.09_{-0.66}^{+0.96}$ & $1.328_{-0.237}^{+0.281}$ & $31.011_{-14.836}^{+42.784}$ \\
$\mathrm{U} 1281$ & 5.5 & 22.7 & 1.7 & 3.2 & 57 & $0.77_{-0.12}^{+0.16}$ & $1.381_{-0.155}^{+0.195}$ & $4.006_{-1.938}^{+7.362}$ \\
$\mathrm{U} 3137$ & 18.4 & 23.2 & 2.0 & 43.6 & 100 & $1.97_{-0.15}^{+0.15}$ & $1.837_{-0.028}^{+0.030}$ & $75.810_{-18.384}^{+117.703}$ \\
$\mathrm{U} 3371$ & 12.8 & 23.3 & 3.1 & 12.2 & 86 & $1.70_{-0.41}^{+0.58}$ & $1.444_{-0.173}^{+0.206}$ & $9.864_{-5.450}^{+26.568}$ \\
$\mathrm{U} 3851$ & 3.4 & 22.6 & 1.5 & 7.3 & 55 & $6.19_{-0.77}^{+0.44}$ & $0.238_{-0.189}^{+0.398}$ & $0.348_{-0.181}^{+0.577}$ \\
$\mathrm{U} 4173$ & 16.8 & 24.3 & 4.5 & 21.2 & 57 & $1.67_{-0.64}^{+0.69}$ & $0.957_{-0.375}^{+0.232}$ & $4.534_{-3.061}^{+12.419}$ \\
$\mathrm{U} 4278$ & 10.5 & 22.5 & 2.3 & 13.6 & 93 & $1.23_{-0.13}^{+0.16}$ & $1.299_{-0.078}^{+0.074}$ & $71.714_{-45.008}^{+112.411}$ \\
$\mathrm{U} 4325$ & 10.1 & 21.6 & 1.6 & 7.5 & 123 & $0.18_{-0.03}^{+0.06}$ & $4.339_{-1.376}^{+3.051}$ & $1.068_{-0.664}^{+2.315}$ \\
$\mathrm{U} 5721$ & 6.7 & 20.2 & 0.5 & 6.6 & 79 & $0.28_{-0.04}^{+0.05}$ & $2.203_{-0.114}^{+0.148}$ & $7.210_{-2.429}^{+12.776}$ \\
$\mathrm{U} 7524$ & 3.5 & 22.2 & 2.3 & 9.7 & 83 & $1.52_{-0.38}^{+1.09}$ & $1.579_{-0.539}^{+0.444}$ & $2.212_{-0.699}^{+0.964}$ \\
$\mathrm{U} 7603$ & 6.8 & 20.8 & 0.7 & 5.4 & 64 & $0.014_{-0.010}^{+0.017}$ & $1.770_{-0.058}^{+0.066}$ & $23.767_{-15.367}^{+105.159}$ \\
$\mathrm{U} 8286$ & 4.8 & 20.9 & 0.8 & 3.5 & 84 & $0.243_{-0.021}^{+0.023}$ & $2.416_{-0.075}^{+0.078}$ & $29.784_{-18.995}^{+77.252}$ \\
$\mathrm{U} 8837$ & 5.1 & 23.2 & 1.2 & 1.6 & 50 & $0.888_{-0.256}^{+0.409}$ & $1.940_{-0.485}^{+0.689}$ & $0.349_{-0.154}^{+0.436}$ \\
$\mathrm{U} 9211$ & 12.6 & 22.6 & 1.2 & 10.5 & 64 & $1.274_{-0.458}^{+0.677}$ & $1.525_{-0.281}^{+0.527}$ & $3.484_{-1.898}^{+8.479}$ \\
$\mathrm{U} 10310$ & 15.6 & 22.0 & 1.9 & 12.6 & 75 & $0.489_{-0.123}^{+0.191}$ & $1.570_{-0.294}^{+0.516}$ & $3.684_{-1.930}^{+6.726}$ \\
\hline & & & & & & & &
\end{tabular}

We can now discuss the obtained values for the stellar mass-to-light ratio. It is easy to see that 10 galaxies have values compatible with the prescribed range by [86]; one (UGC3851) has a higher value which is hard to explain in term of reasonable population synthesis; and four (UGC4325, UGC5721, UGC7603, UGC8286) have values smaller than 0.5. Among these, one is particularly problematic, i.e. UGC7603, because it has a mass-to-light ratio too much small, $Y_{*}=0.017$, while the others can be again considered acceptable.

UGC3851 is a real challenge for both our approach and the more traditional one [31]. The very linear rise in the inner region rapidly changes in a flat part at larger radii so that it is very hard to reproduce this sharp change in the slope. There are two possibilities for this behavior: the HI curve in outer points is underestimated, or there are non-circular motions related to a bar-like structure and a star forming region in the center which affect the $\mathrm{H} \alpha$ observations. It is interesting to note the many similarities between our analysis and that one in 31]: both their NFW and isothermal halo model overestimate velocity in the inner region, with a larger deviation in the NFW model than the isothermal one, and larger in the inner region than in the outer one, as in our case. Then their derived maximum value for the stellar mass-to-light ratio is $Y_{*}=5.4$, very close to our value. Anyway, all the following relations will be derived without considering this galaxy because no definitive conclusion can be done about it.

Among the four galaxies with a low mass to light ratio, one of the most problematic case is UGC5721, showing a very narrow bi-modality in the parameters distribution that cannot be avoided and resolved even changing parameters of the trial distribution in the MCMC. The convergence test inevitably fails in this case, but we have assumed as best fit results the values associated to the highest peak in the likelihood distribution shown in Fig. (9), which produce a very good fit to the observational rotation curve. A discrepancy can be found around $2 \mathrm{kpc}$ but as the large error bar shows, in this region there are some observational difficulties related to gas observations which produce the detected faster rise in velocity.

For what concerning the scalar field parameters, the coupling constant $\beta$ seems to be very well constrained in the range $[0.957 ; 2.416]$, if we exclude the previously discussed case of UGC3851, whose value is very low, $\beta=0.238$, probably because of the degeneracy with $Y_{*}$, and the opposite situation of UGC4325, which exhibits a large value, $\beta=4.339$, and also larger errors on this parameter than other galaxies. It is not a causality that this is also one of the four galaxies with a stellar massto-light ratio lower than the simulation limit.

As in clusters case, the scalar field length shows a larger 

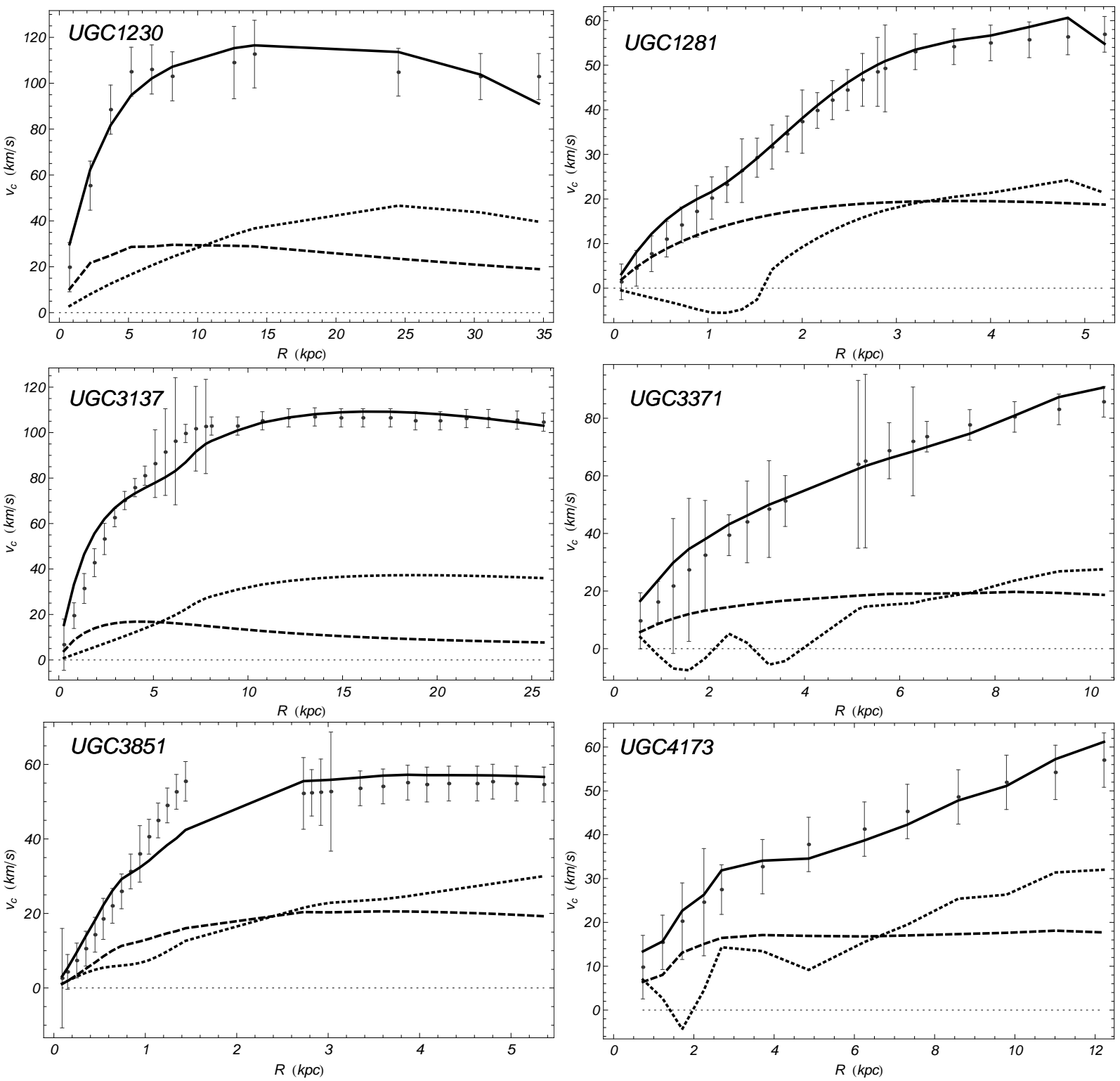

Figure 7. Rotation curves of LSB galaxies. Dots are velocities from data; solid line is the theoretical model, Eq. (69); dashed line is the stellar contribution to the rotation curve assuming $Y_{*}=1$; dotted line is the gas contribution.

spread, mainly scaling with the dimension of any LSB galaxy. One problematic situation is UGC8837, which clearly deviates from the general trend for what concerning the value of scalar field length $L$, resulting it very small. Even if the fit of our model to data is very good, we do not consider anymore this galaxy in the following discussion because its results are strongly related to the low quality of data. As discussed in [31], the $\mathrm{H} \alpha$ are of good quality, but do not correspond very well to the HI profile, with a large difference in their systemic velocity probably due to the interference of inner star formation region and there located non-circular motions. A further error source can be also the inclination, being this galaxy almost edge-on, the most problematic configuration in this case.
Just by visual inspection it is possible to see that the general shapes of the rotation curves are quite well predicted by our model inside the error confidence region, even considering some singular cases where are present irregularities coming from gas distribution (clumpy peaks, change in the profile convexity). In evaluating the goodness of our fitted parameters we have to note that all the rotation curves are limited to a certain distance from the center of any galaxy. It is well known that spiral galaxies show a great variety of rotation curves profiles, with flat velocity plateaux, different slopes (both increasing and decreasing) in outer regions; but in our case the necessity to match stellar photometry with more extended gas observations, results in a limited distance range which may affect our 

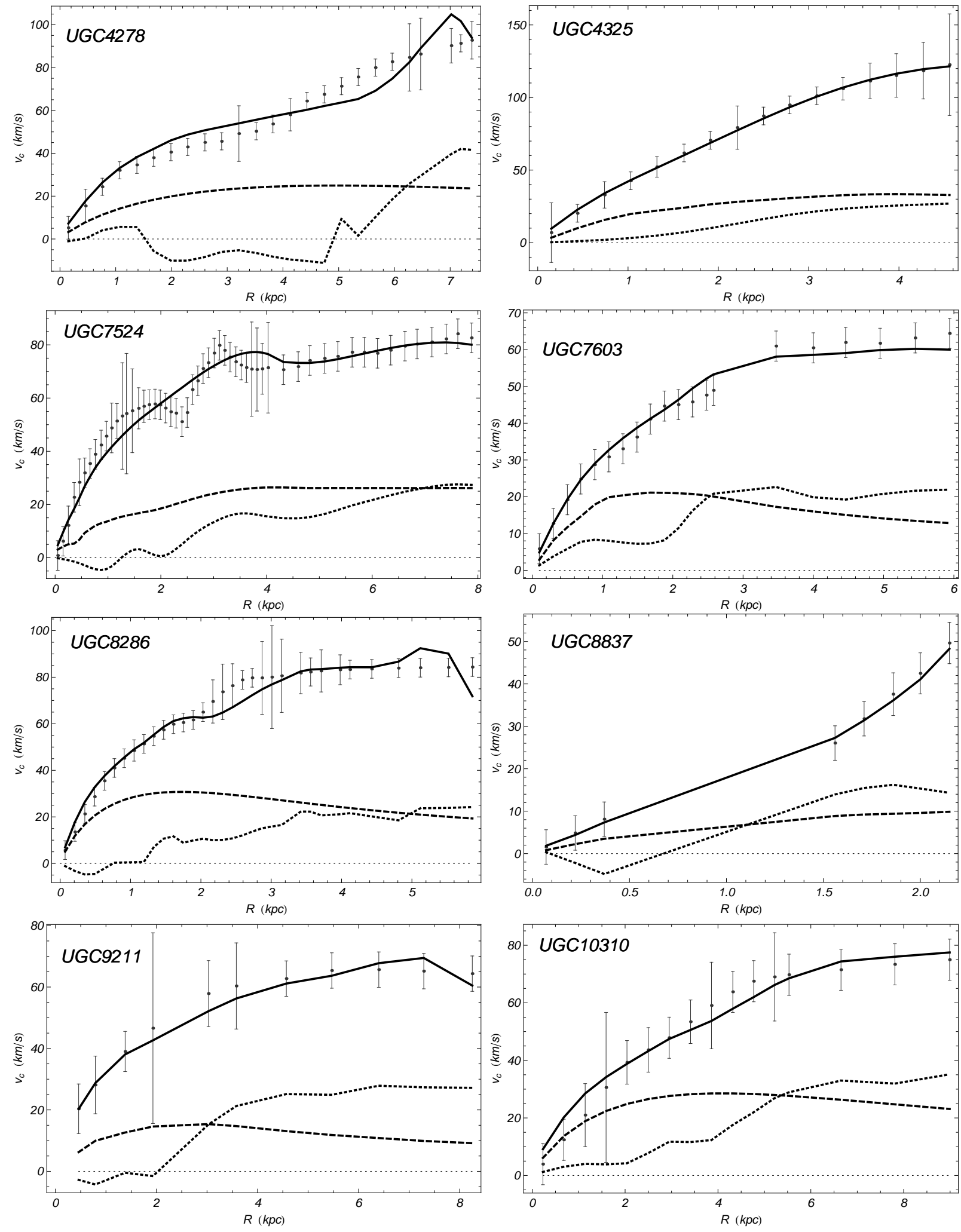

Figure 8. Same of Fig. (77). 


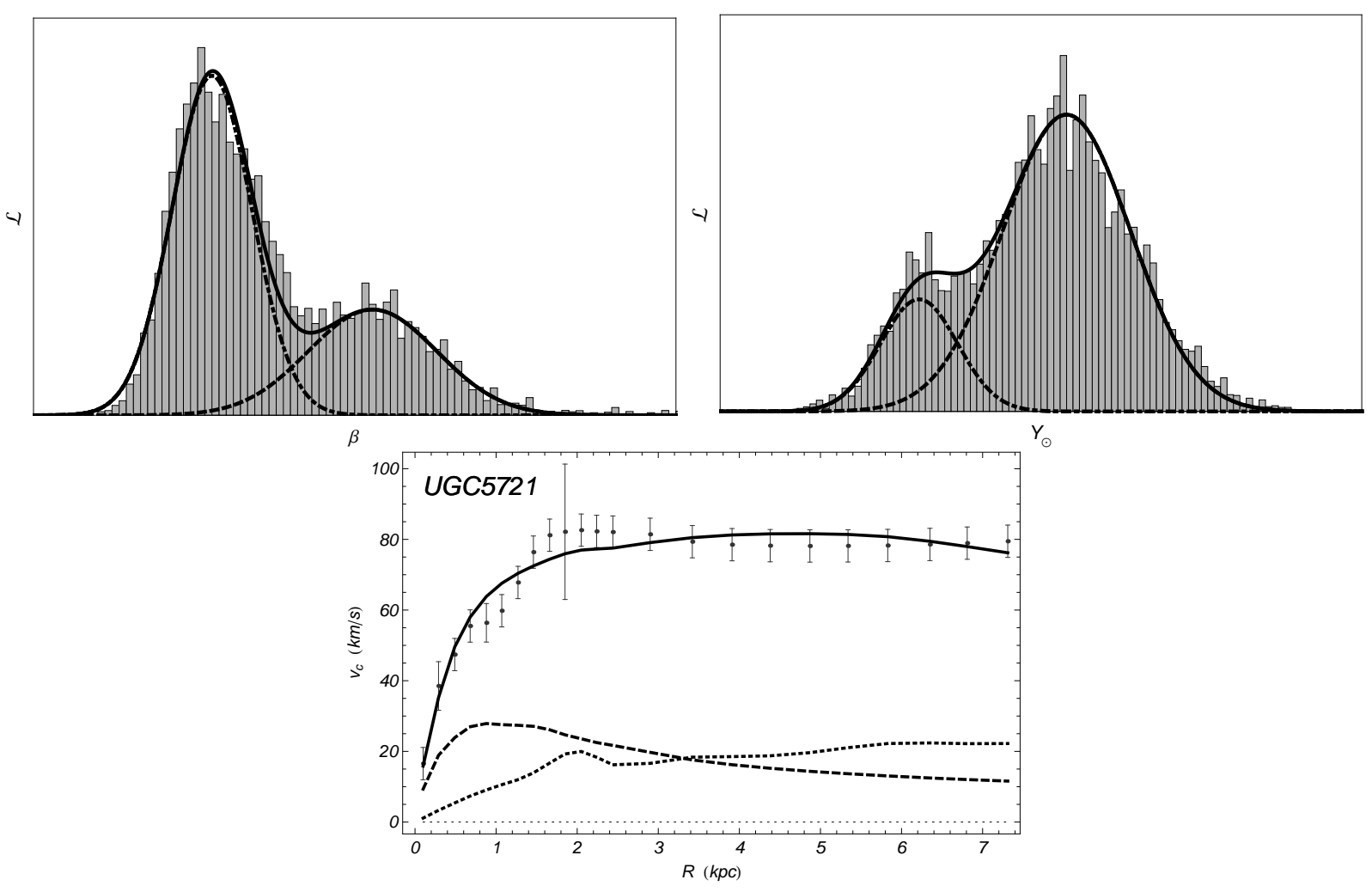

Figure 9. Same of Fig. (7) for UGC5721. In upper plots are shown the marginalized probability distributions for $\beta$ and $Y_{*}$ obtained from MCMCs and the unresolved bi-modality discussed in $\S$ (IVC).

results. As pointed out in [18] this could not solve the previously described degeneracies between the fitting parameters, or to induce their wrong estimations, so requiring more extended data for a more exact analysis.

Then, for best appreciating the statistical validity of our analysis, we can compare them with a recent work [81] on the same class of galaxies but based on MOND as alternative theoretical model to dark matter. MOND is well-know to be the main and most successful alternative scenario in explaining rotation curves of spiral galaxies, even if not being satisfactory (when even completely unable) in describing mass profiles in clusters of galaxies. In 81 MOND results to predict quite well LSB rotation curves; a look to their figures show that even MOND is unable to explain all the features that appear in such profiles, and even some open questions remain, that seem to be intrinsic in the model while only weakly depending on possible observational sources of uncertainties.

A positive step of our model, is that we are able to obtain sufficiently good fits for two very different gravitational systems as clusters of galaxies and LSB and opening at the same time the possibility of a unique theoretical background underlying both them.

Moreover, some of the correlations they find between MOND acceleration and physical properties of LSB galaxies can be found in our approach too. For example, we find a correlation among the parameter $\beta$ and the ex- trapolated central disk surface brightness $\mu_{0, R}$, even more general than their on, because in our case it well fit all the LSB galaxies, without any cut-of dependence (their correlation strongly depends on galaxies with higher surface densities). Two possible fits are:

$$
\beta=-0.277167 \cdot \mu_{0, R}+7.78297
$$

and

$$
\log \beta=-3.80827 \cdot \log \mu_{0, R}+5.32519
$$

and, as shown in the top panel of Fig. (10), they are almost indistinguishable. Then, we also have a correlation among both scalar field parameters and the maximum rotation velocity, while in MOND no correlation has been found. In the middle and bottom panels of Fig. (10) we have:

$$
\begin{gathered}
\log \beta=0.354674 \cdot \log V_{\max }-0.475189 \text { and } \\
\log L=3.81376 \cdot \log V_{\max }-6.14695 .
\end{gathered}
$$

Still some questions have to be better studied, because for LSB galaxies we could not have performed a detailed analysis of scaling or structural parameters as we did with clusters of galaxies.

Anyway, the selected sample has given important details and perspectives about the possibility that scalar 

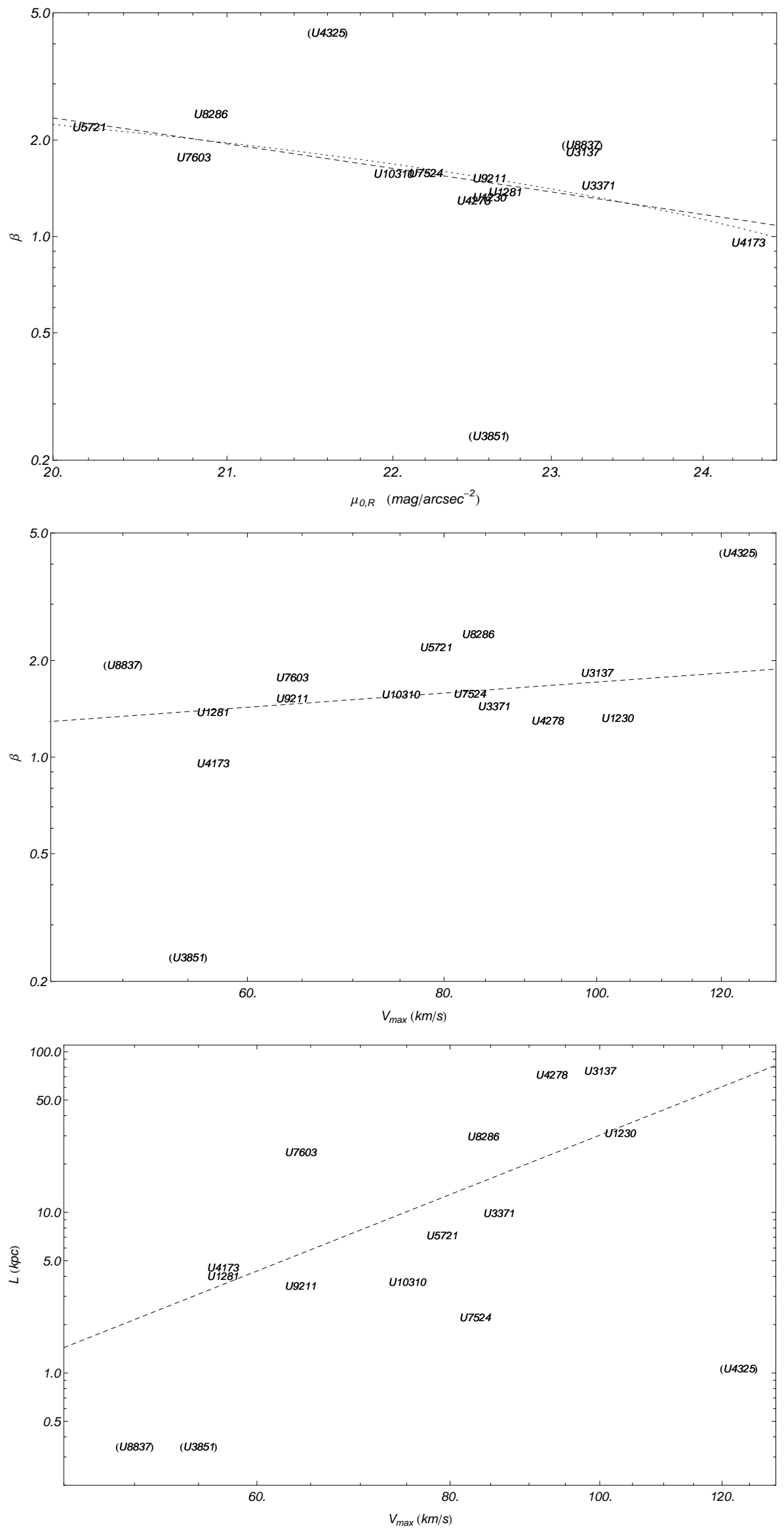

Figure 10. Correlations among scalar field parameters and structural properties of LSB galaxies. (Top panel.) Coupling constant $\beta$ versus the central disk surface brightness. The dotted line is the linear fit; the dashed line is the log-log fit. (Middle panel.) Coupling constant $\beta$ versus the maximum rotation velocity. (Bottom panel.) Scalar field length $L$ versus the maximum rotation velocity. Objects in brackets are excluded from fits as described in $\S$ (IV). 
field works well even at these scales. But the large dispersion in scalar field parameters show that something more accurate should be done. The used sample of LSB galaxies is limited by the fact that many rotation curves are not smooth, not extended to large radii; and then they constitute a limited sample of a more complex and extended class of gravitational systems as galaxies. In a forthcoming paper we are going to revisit all these questions, enlarging the galaxy sample from dwarf and irregular galaxies, to high surface brightness (HSB) spiral galaxies, and to elliptical galaxies.

\section{Unified picture}

Even with all the previous caveats in mind, it seems that results are consistent when we compare clusters and LSB analysis. We can say more: even if we will only derive phenomenological and visual relations, whose physical meaning has to be studied in more detail, they can help us in finding problematic cases and thus verifying $a$ posteriori if they are such for intrinsic problems of our model or for something different.

Figs. (11) - (15) show that a possible general trend including both clusters and LSB galaxies is feasible.

In Fig. (11) a correlation between the scalar field length $L$ and the gas density of any object is possible. We have considered only gas contribution, because we do not want to take into account dark matter and use only visible matter: in clusters of galaxies, gas is largely the main contribution to the visible mass; and for a self-consistent discussion, we have considered an analogous quantity for LSB galaxies too, also considering that for calculating the stellar mass contribution, we have to use the massto-luminosity ratio $Y_{*}$, being this a parameter fit.

For clusters (without the problematic cases: Abell 2390, MKW4, RXJ1159) we have:

$$
\log L=-1.56605 \cdot \log \rho_{\text {gas }}+8.99653 ;
$$

for LSB galaxies (without UGC3851, UGC4325, UGC8837):

$$
\log L=-1.8617 \cdot \log \rho_{\text {gas }}+9.95973 ;
$$

while for the total sample (without the previous exceptions):

$$
\log L=-1.85764 \cdot \log \rho_{\text {gas }}+10.1326
$$

We can note that a small difference in the slope between the total and the cluster-only sample, while the one from spiral is practically equivalent. This may lead us to say that a possible general trend is present, and that, likely, it can be made more clear when adding further intermediate data, as group of galaxies and elliptical galaxies, or smaller scales ones.

The same, but for the coupling constant $\beta$, is shown in Fig. (12). For clusters (without Abell 2390, MKW4,
RXJ1159) it is:

$$
\log \beta=0.0946259 \cdot \log \rho_{\text {gas }}-0.0237813 ;
$$

for LSB galaxies (without UGC3851, UGC4325, UGC8837):

$$
\log \beta=0.446629 \cdot \log \rho_{\text {gas }}-1.92872 ;
$$

and for the total sample (without the previous exceptions):

$$
\log \beta=-0.0290609 \cdot \log \rho_{\text {gas }}+0.444376
$$

In this case we see that the total-sample slope is different from the other two cases, even if the one from the cluster-only sample is very small, and also by visual inspection clusters seem to be less spread around the main general relation than LSB galaxies. These last ones, on the contrary, seem to show a proper intrinsic slope, even if it can depend on the previously described problems we are facing with when working with a restricted galaxy sample, or with not enough extended rotation curves.

We note that it is much important to verify a very low value $(\approx 0)$ for $\beta$ because we remind that in $\oint$ II we assumed that $\beta$ is constant or at least has a weak dependence on scale. This hypothesis is partially confirmed by clusters and by the total sample fit.

The same conclusion can be derived from Fig. (13) where we plot $\beta$ versus $L$ : it is evident that there is quite no dependence for $\beta$ on the gravitational scale. In this case we also note that the global fit feels more clusters than LSB, having performed a weighted fit and clusters showing best constraints on scalar field parameters. For clusters (without Abell 2390, MKW4, RXJ1159) we have:

$$
\log \beta=-0.11781 \cdot \log L+0.697316 ;
$$

for LSB galaxies (without UGC3851, UGC4325, UGC8837):

$$
\log \beta=-0.00355508 \cdot \log L+0.197588 ;
$$

and for the total sample (without the previous exceptions):

$$
\log \beta=0.0176545 \cdot \log L+0.306458
$$

When plotting the scalar field parameters versus the total gas mass enclosed in the considered gravitational structures it is more evident the need of more objects for giving more detailed and best constrained results. In Figs. (14) - (15) it is evident the big void lying among clusters and LSB regions.

We have given possible fits even in this case; for clusters (without Abell 2390, MKW4, RXJ1159) we have:

$$
\begin{gathered}
\log L=0.558047 \cdot \log \frac{M_{\text {gas }}}{10^{9} M_{\odot}}+0.273985, \\
\log \beta=-0.0745605 \cdot \log \frac{M_{\text {gas }}}{10^{9} M_{\odot}}+0.702031 ;
\end{gathered}
$$




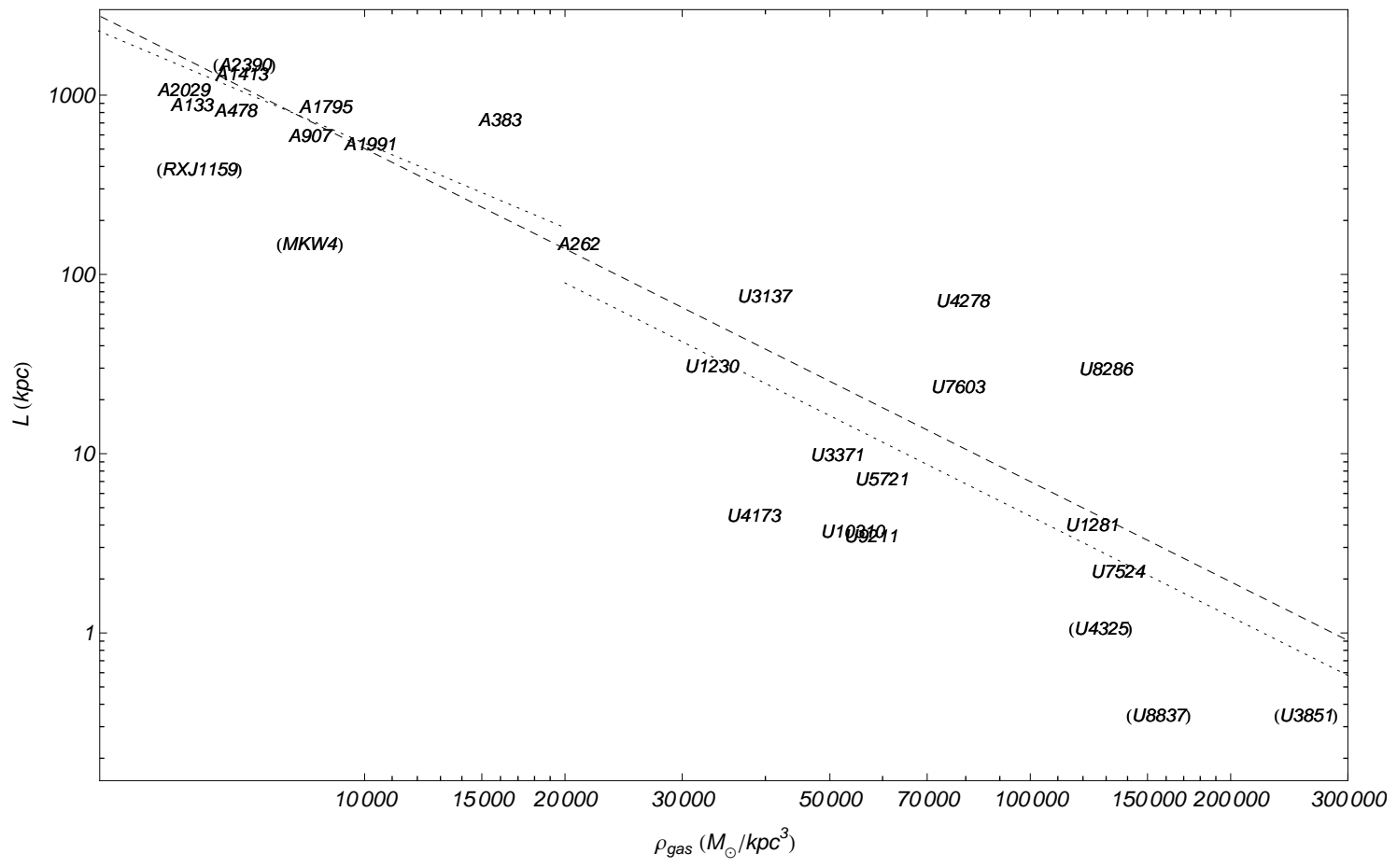

Figure 11. $L$ vs $\rho_{\text {gas }}$. The dotted lines are the singular fits to clusters and LSB samples. The dashed line is the fit to the total (clusters + LSB) sample. The fits are weighted with errors on parameters derived from MCMCs. Objects in brackets are excluded from fits as described in $\S$ (IV).

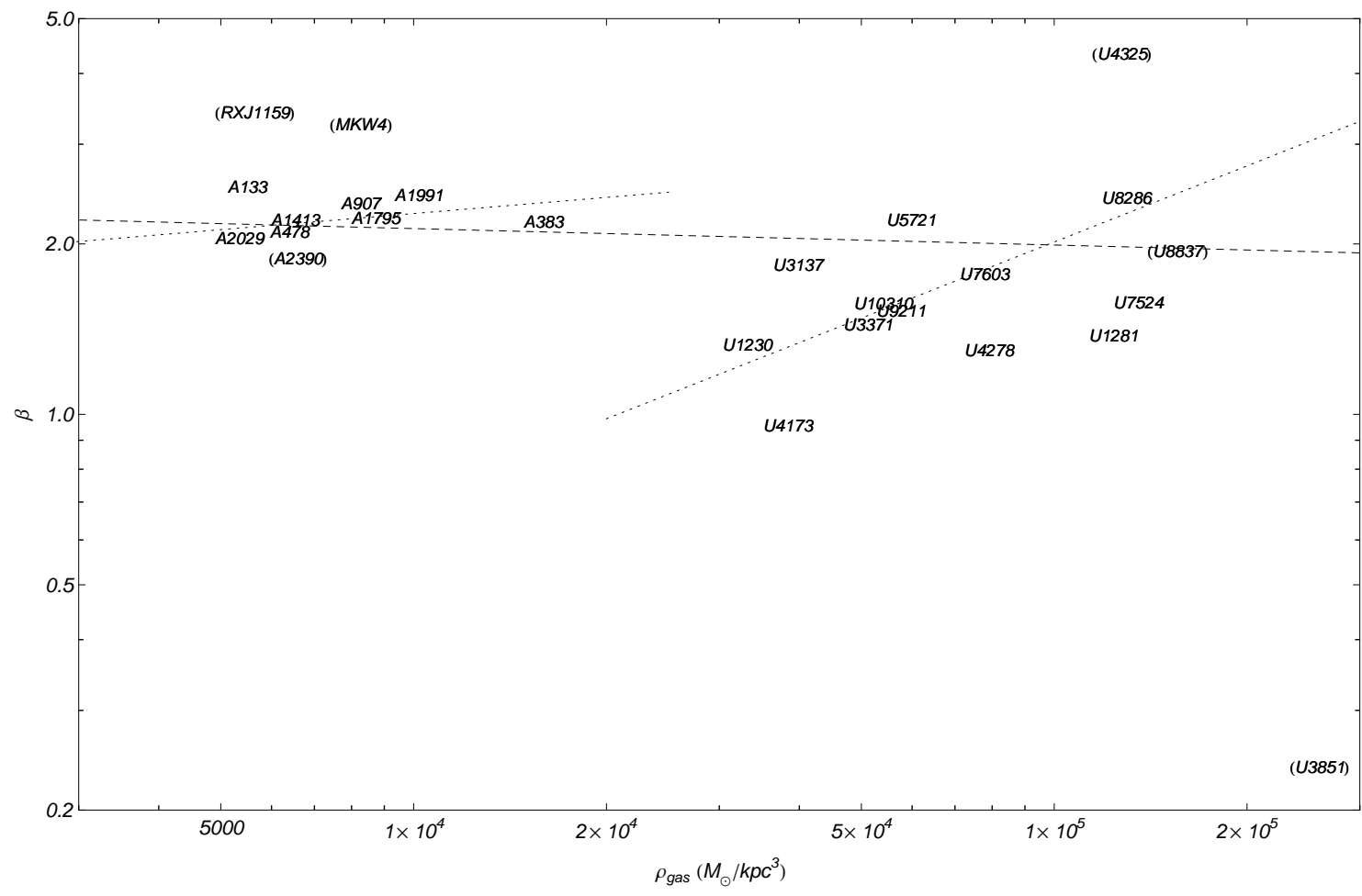

Figure 12. $\beta$ vs $\rho_{\text {gas }}$. The dotted lines are the singular fits to clusters and LSB samples. The dashed line is the fit to the total (clusters + LSB) sample. The fits are weighted with errors on parameters derived from MCMCs. Objects in brackets are excluded from fits as described in $\S$ (IV). 


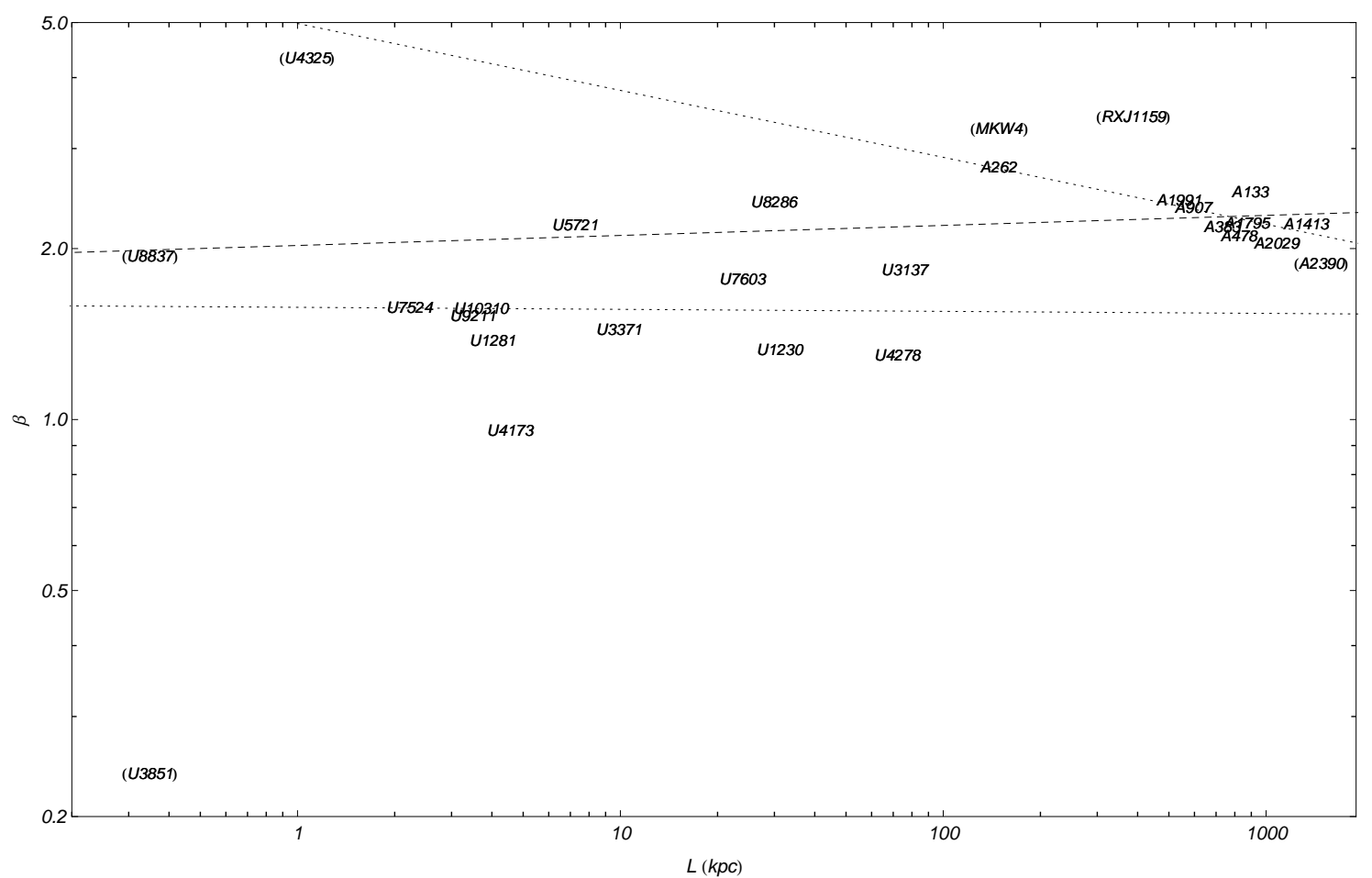

Figure 13. $\beta$ vs $L$. The dotted lines are the singular fits to clusters and LSB samples. The dashed line is the fit to the total (clusters + LSB) sample. The fits are weighted with errors on parameters derived from MCMCs. Objects in brackets are excluded from fits as described in $\S$ (IV).

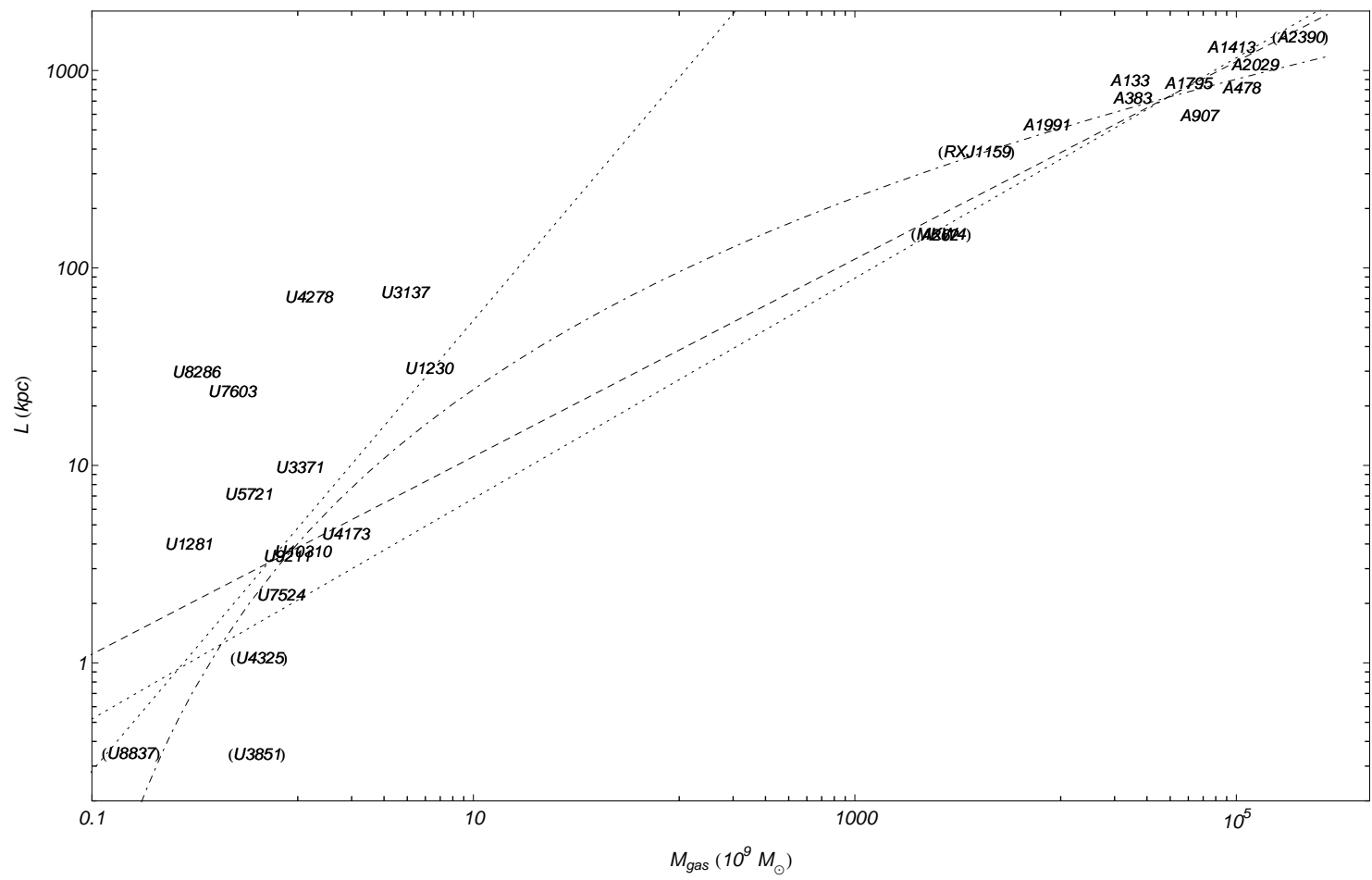

Figure 14. $L$ vs $M_{\text {gas }}$. The dotted lines are the singular fit to clusters and LSB samples. The dashed line is the linear fit to the total (clusters + LSB) sample. The dot-dashed line is the logarithmic fit to the total (clusters + LSB) sample. The fits are weighted with errors on parameters derived from MCMCs. Objects in brackets are excluded from fits as described in $\S$ (IV). 


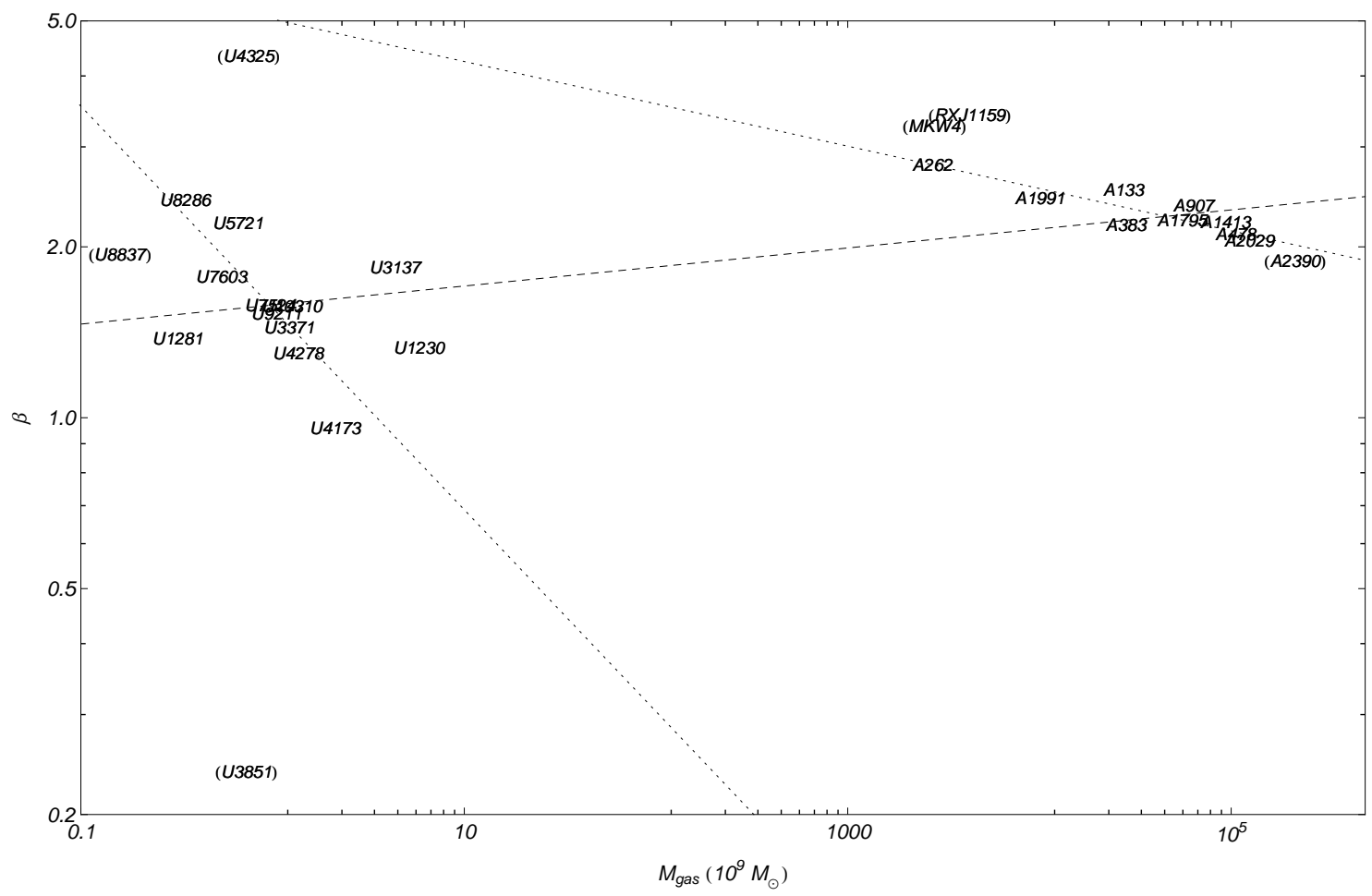

Figure 15. $\beta$ vs $M_{\text {gas }}$. The dotted lines are the singular fit to clusters and LSB samples. The dashed line is the fit to the total (clusters + LSB) sample. The fits are weighted with errors on parameters derived from MCMCs. Objects in brackets are excluded from fits as described in $\S$ (IV).

while for LSB galaxies (without UGC3851, UGC4325, UGC8837):

$$
\begin{array}{r}
\log L=1.141 \cdot \log \frac{M_{\text {gas }}}{10^{9} M_{\odot}}+0.592915, \\
\log \beta=-0.355447 \cdot \log \frac{M_{\text {gas }}}{10^{9} M_{\odot}}+0.193349 ;
\end{array}
$$

For the total sample (without the previous exceptions) we have tried two different fits for the length $L$ :

$$
\log L=1.141 \cdot \log \frac{M_{\text {gas }}}{10^{9} M_{\odot}}+0.592915,
$$

$$
\log L=3.67182 \cdot \log \left(1.38315 \log \frac{M_{\text {gas }}}{10^{9} M_{\odot}}\right)+1.59465,
$$

but without intermediate data we cannot infer any conclusion; while for $\beta$ we have:

$$
\log \beta=0.0335265 \cdot \log \frac{M_{\text {gas }}}{10^{9} M_{\odot}}+0.198459 .
$$

\section{CONCLUSIONS}

In this work we studied the dynamical properties of several astrophysical systems within the theoretical framework of scalar theories. We investigate whether there are evidences for a scalar field in the considered astrophysical systems and if it is possible to observationally detect it. We have taken into account three different classes of objects: supernovae, low surface brightness spiral galaxies and clusters of galaxies. Results show that: $i)$ there is an intrinsic difficulty in extracting information about scalar field mechanism (or more generally about a varying gravitational constant) from supernovae; $i$ ) a scalar field can fairly well reproduce the matter profile in clusters of galaxies, estimated by X-ray observations and without the need of any additional dark matter; $i i i$ ) good fits to the rotation curves of low surface brightness galaxies, using visible stellar and gas mass components, are obtained.

These results show that different astrophysical system can be used as different tracers of the same physical mechanism. Moreover, they point towards the possibility of a unifying view of dark matter and dark energy via a scalar field, at least at galactic and cluster scales [22]. The main criticism of the approach is related to the fact that the very different physical properties and evolution of the considered astrophysical systems could insert unwanted biases and priors leading to a wrong overall picture of the underlying cosmological model. This shortcoming could be partially avoided if homogeneous and well calibrated samples of data at low, medium and high redshifts will be achieved in future. 


\section{ACKNOWLEDGMENTS}

DFM thanks the Research Council of Norway FRINAT grant 197251/V30 and the Abel extraordinary chair UCM-EEA-ABEL-03-2010. DFM is also partially supported by the projects $\mathrm{CERN} / \mathrm{FP} / 109381 / 2009$ and PTDC/FIS/102742/2008. VS has been partially funded by the Research Council of Norway with a fellow un- der the YGGDRASIL programme 2009-2010 and is now working at UPV/EHU under the project "Convocatoria para la concesión de Ayuda a la Especialización para Investigadores Doctores en la UPV/EHU-2009". SC acknowledges the support of INFN (Sez. di Napoli) and the ERASMUS/SOCRATES European program. VS acknowledges V. F. Cardone for helpful comments and suggestions.
[1] Astier, P. et al. 2006, A\&A, 447, 31

[2] Bahcall, N. A., in "Formation of structure in the universe", 1995, Jerusalem Winter School, astro-ph/9611148

[3] Bahcall, N.A. et al. 2003, Astrophys. J., 585, 182

[4] Bahcall, N.A., Bode, P. 2003, Astrophys. J., 588, 1

[5] Binetruy P.,2006, Supersymmetry: Theory, experiment and cosmology, Oxford, UK: Oxford Univ. Pr. $520 \mathrm{p}$

[6] Binney, J., Tremaine, S., in "Galactic Dynamics", 1987, Princeton University Books, Princeton (USA)

[7] Brax, P., van de Bruck, C., Davis, A.-C., Khoury, J., Weltman, A., 2004, Phys. Rev. D, 70, 123518

[8] Brax P., van de Bruck C., Davis A. and Green A., (2006) Phys. Lett. B 633, 441

[9] Brax P., van de Bruck C., Davis A.C. , Mota D.F. and Shaw D.J.,(2007a) Phys. Rev. D 76, 124034

[10] Brax P., van de Bruck C., Davis A., Mota D.F. and Shaw D.J.,(2007b) Phys. Rev. D 76, 085010

[11] Brax P., van de Bruck C., Davis A. and Shaw D.,(2008) Phys. Rev. D 78, 104021

[12] Bourliot F., Ferreira P., Mota D.F. and Skordis C., (2007) Phys. Rev. D 75, 063508

[13] Brownstein, J. R., Moffat, J. W., 2006, MNRAS, 367, 527

[14] Bryan, G.L., Astrophys. J., 495, 80

[15] Bueno Sanchez, J.C., Nesseris, S., Perivolaropoulos, L., 2009, JCAP, 11, 29

[16] Carroll, S.M., Press, W.H., Turner, E.L. 1992, ARA\&A, 30, 499

[17] Capozziello, S., 2002, Int. Jou. Mod. Phys. D 11, 483

[18] Capozziello, S., Cardone, V.F., Troisi, A., 2007, MNRAS, 375, 1423-1440

[19] Capozziello S. and Tsujikawa S.,(2008) Phys. Rev. D 77, 107501

[20] Capozziello, S. and Francaviglia, M.,(2008) Gen. Rel. Grav. 40, 357

[21] Capozziello, S., de Filippis, E., Salzano, V., 2009, MNRAS, 394, 947.

[22] Cardone, V.F., Troisi, A., Capozziello, S.; 2005 Phys. Rev. D 72, 043501.

[23] Chakrabarty, D., de Filippis, E.,\& Russell, H. 2008, A\&A, 487, 75

[24] Chevallier, M., Polarski, D., 2001, Int. J. Mod. Phys. D 10, 213

[25] Clocchiati, A. et al. 2006, Astrophys. J., 642, 1

[26] Copeland E.J., Sami M., Tsujikawa S., 2006, Int. J. Mod. Phys. D 15, 1753

[27] Cole, S. et al. 2005, MNRAS, 362, 505

[28] Croft, R.A.C., Hu, W., Dave, R. 1999, Phys. Rev. Lett., 83, 1092

[29] de Bernardis, P. et al. 2000, Nature (London), 404, 955
[30] de Blok, W.J.G., McGaugh, S.S., 1997, MNRAS, 290, 533

[31] de Blok, W.J.G., Bosma, A., 2002, A\&A, 385, 816

[32] De Filippis, E., Sereno, M., Bautz, Longo G., M. W., 2005, Astrophys. J., 625, 108

[33] Davis A.C., Schelpe C. and Shaw D., (2009) Phys. Rev. D 80, 064016

[34] Dunkley, J., Bucher, M., Ferreira, P. G., Moodley, K., Skordis, C., 2005, MNRAS, 356, 925

[35] Eisenstein, D. et al. 2005, Astrophys. J., 633, 560

[36] Eke, V.R., Cole, S., Frenk, C.S., Petrick, H.J. 1998, MNRAS, 298, 1145

[37] Esposito-Farese, G., Polarski, D., Phys. Rev. D, 63, 2001, 063504

[38] Evrard, A.E., Metzler, C.A., Navarro, J.F., 1996, Astrophys. J., 469-494

[39] Faulkner T., Tegmark M., Bunn E. and Mao Y.,(2007) Phys. Rev. D 76, 063505

[40] Feldman B. and Nelson A., (2006) JHEP 0608, 002

[41] Freeman, K., 1970, Astrophys. J., 160, 811

[42] Gannouji, R., Moraes, B., Polarski, D., arXiv:0907.0393

[43] Gies H., Mota D.F. and Shaw D.J., (2008) Phys. Rev. D 77, 025016

[44] Hicken, M., et al., 2009, Astrophys. J., 700, 331-357

[45] Hicken, M., Wood-Vasey, W. M., Blondin, S., Challis, P., Jha, S., Kelly, P. L., Rest, A., Kirshner, R. P., 2009, Astrophys. J., 700, 1097-1140

[46] Ito Y, and Nojiri S., (2009) Phys. Rev. D 79, 103008

[47] Jeffreys, H., 1961, Theory of Probability, Oxford University Press, Oxford, 3rd ed.F

[48] Khoury, J., Weltman, A., 2004, Phys. Rev. D, 69, 044026

[49] Kowalski, M., Rubin, D., Aldering, G. et al., 2008, Astrophys. J., 686, 749

[50] Komatsu, E., Smith, K.M., Dunkley, J., Bennett, C.L., Gold, B., Hinshaw, G., Jarosik, N., Larson, D., Nolta, M.R., Page, L., Spergel, D.N., Halpern, M., Hill, R.S., Kogut, A., Limon, M., Meyer, S.S., Odegard, N., Tucker, G.S., Weiland, J.L., Wollack, E., Wright, E.L., arXiv:1001.4538, submitted to ApJS

[51] Linder, E.V., 2003, Phys. Rev. Lett., 90, 091301

[52] Linde A.D., 2008 , Lect. Notes Phys. 738, 1

[53] McDonald, P. et al. 2005, Astrophys. J., 635, 761

[54] McGaugh, S.S., de Blok, W.J.G., 1998, Astrophys. J., 499, 41

[55] Moore, B., 1994, Nature (London), 370, 629

[56] Navarro, J.F., Frenk, C.S., White, S.D.M., 1996, Astrophys. J., 462, 563

[57] Mota D.F., (2008) JCAP 0809006

[58] Mota D.F. and Barrow J.D.,(2004) Phys. Lett. B 581, 141 
[59] Mota D.F. and Shaw D.J.,(2006) Phys. Rev. Lett. 97, 151102

[60] Mota D.F. and Shaw D.J., (2007) Phys. Rev. D 75 063501

[61] Mota D.F., Pettorino V., Robbers G. and Wetterich C., (2008) Phys. Lett. B 663, 160

[62] Mota D.F., Kristiansen J., Koivisto T. and Groeneboom N., (2007) Mon. Not. Roy. Astron. Soc. 382, 793

[63] Neumann., D. M., Böhringer, H., 1995, A\&A, 301, 865

[64] O'Sullivan, E., vrtilek, J.M., Read, A.M., David, L.P., Ponman, T.J., 2003, MNRAS, 346, 525

[65] Padmanabhan, T. 2003, Phys. Rep., 380, 235

[66] Peebles, P.J.E., Rathra, B. 2003, Rev. Mod. Phys., 75, 559

[67] Perlmutter, S., 1999 Astrophys. J., 517, 565-586

[68] Pope, A.C. et al. 2005, Astrophys. J., 607, 655

[69] Refregier, A. 2003, ARA\&A, 41, 645

[70] Riazuelo, A., Uzan, J.-P., 2002, Phys. Rev. D, 66, 023525

[71] Riess, A.G. et al. 2004, Astrophys. J., 607, 665

[72] Sahni, V., Starobinski, A. 2000, Int. J. Mod. Phys. D, 9, 373

[73] Sanchez, A.G. et al. 2006, MNRAS, 366, 189

[74] Schindler, S., 2004, Ap\&SS, 28, 419

[75] Schmidt, R. W., Allen S. W., 2007, MNRAS, 379, 209

[76] Schwarz, G., 1978, Annals of Statistics, 5, 461

[77] Seljak, U. et al. 2005, Phys. Rev. D, 71, 103515
[78] Spergel, D.N. et al. 2003, ApJS, 148, 175

[79] Swaters, R.A., Ph.D. Thesis, University of Leiden

[80] Swaters, R.A., Madore, B.F., Trewhella, M., 2000, Astrophys. J., 531, L107

[81] Swaters, R.A., Sanders, R.H., McGaugh, S.S., 2010, Astrophys. J., 718, 380

[82] Tegmark, M. et al. 2004, Phys. Rev. D, 69, 103501

[83] Tamaki T. and Tsujikawa S., (2008) Phys. Rev. D 78, 084028

[84] Tsujikawa S., Tamaki T. and Tavakol R.,(2009) JCAP 0905020

[85] Upadhye A., Steffen J.H., Weltman A., (2010) Phys. Rev. D 81, 015013

[86] van den Hoek, L.B., de Blok, W.J.G., van der Hulst, J.M., de Jong, T., 2000, A\&A, 357, 397

[87] van Waerbecke, L. et al. 2001, A\&A, 374, 757

[88] Viana, P.T.P., Nichol, R.C., Liddle, A.R. 2002, Astrophys. J., 569, 75

[89] Vikhlinin, A., Markevitch, M., Murray, S. S., Jones, C., Forman, W., Van Speybroeck, L., 2005, Astrophys. J., 628,655

[90] Vikhlinin, A., Kravtsov, A., Forman, W., Jones, C., Markevitch, M., Murray, S. S., Van Speybroeck, L., 2006, Astrophys. J., 640, 691

[91] Voit, G.M., 2005, Rev. of Mod. Phys., 77, 207

[92] Zwicky, F., 1933, Helv. Phys. Acta, 6, 110 